Research Article

\title{
KDM1 A Identified as a Potential Oncogenic Driver and Prognostic Biomarker via Multi-Omics Analysis
}

\author{
Lingyue Li, ${ }^{1}$ Yiyu Wang, ${ }^{1}$ Yuan Mou, ${ }^{1}$ Hao Wu $\mathbb{D}^{2},{ }^{2}$ and Ye Qin $\mathbb{D D}^{1}$ \\ ${ }^{1}$ Hubei Key Laboratory of Tumor Microenvironment and Immunotherapy, China Three Gorges University, Yichang 443002, \\ Hubei Province, China \\ ${ }^{2}$ Department of Oncology, The First Affiliated Hospital of Nanjing Medical University, Nanjing 210029, Jiangsu Province, China
}

Correspondence should be addressed to Hao Wu; whdactor@163.com and Ye Qin; ycqinye@163.com

Received 3 October 2021; Accepted 12 November 2021; Published 9 December 2021

Academic Editor: Meng-Hao Huang

Copyright (C) 2021 Lingyue Li et al. This is an open access article distributed under the Creative Commons Attribution License, which permits unrestricted use, distribution, and reproduction in any medium, provided the original work is properly cited.

\begin{abstract}
Background. Lysine-specific demethylase 1A (KDM1A) is a histone demethylation enzyme and a crucial epigenetic factor for multiple pathological pathways that mediate carcinogenesis and immunogenicity. Although increasing evidence supposes the association between KDM1A and cancers, no systematic multi-omics analysis of KDM1A is available. Methods. We systematically evaluated the KDM1A expression of various cancer and normal tissues and the unique relationship between KDM1A expression and prognosis of cancer cases based on The Cancer Genome Atlas (TCGA), Genotype Tissue Expression (GTEx), and Clinical Proteomic Tumor Analysis Consortium (CPTAC) database. The genetic variations, phosphorylation, and DNA methylation of KDM1A were analyzed via various tools. We further analyzed the correlation of KDM1A expression and fibroblasts and immune cell infiltration score of TCGA samples via TIMER2.0. Results. KDM1A was highly expressed in 17 types of total 33 cancers, while it expressed low levels in only 4 cancers. High KDM1A expression was associated with worse survival status in various cancers. KDM1A expression was positively correlated with the cancer-associated fibroblasts and myeloid-derived suppressor cells infiltration levels in most cancer types. Additionally, KDM1A in most cancer types was negatively correlated with Th1 cell infiltration and positively correlated with Th2 cells. Moreover, spliceosome, cell cycle, and RNA transport pathways were involved in the functional mechanisms of KDM1A via enrichment analysis. Conclusions. Our study describes the epigenetic factor KDM1A as an oncogene and prognostic biomarker. Our findings provide valuable guidance for further analysis of KDM1A function in pathogenesis and potential clinical treatment.
\end{abstract}

\section{Introduction}

Epigenetics has been proved as one of the fundamental mechanisms leading towards carcinogenesis [1]. The irregularities of the epigenome associated with cancer are regulated via histone modifications, DNA methylation, chromatin remodeling, and stability of RNA transcripts. The advancement in genomic technologies over the last two decades provided us with a bird's eye view of the epigenetic factors in oncogenesis, including oncogenic and tumorsuppressor networks. Moreover, the epigenetic changes in cancer cells exposed a key role in the effects of tumor-host interactions, especially with immune cells and stromal cells [2]. With improved understanding, epigenetic modifications in cancer are possibly reversible, indicating that epigenetic regulation is a promising therapeutic target to explore.

The lysine-specific demethylase 1A (KDM1A), also known as LSD1or AOF2, was the first histone demethylation enzyme identified by Shi et al. [3]. It revealed the dynamic regulation of histone methylation by both histone methylases and demethylases. KDM1A has been shown to demethylate histone $\mathrm{H} 3$ on lysine 4 (H3K4) and lysine 9 (H3K9), which functions in the regulation of gene expression as a transcriptional repressor or activator [3, 4]. Furthermore, a neuron-specific isoform of LSD1 (KDM1A), LSD1n, was described to acquire a new substrate specificity targeting H4K20me2 methylation for transcription activation of neuronal-regulated genes [5]. The expression of KDM1A has 
been found upregulated and correlated with poor prognosis in various cancer types [6-9]. KDM1A plays a pivotal role in various cancer-related physiological processes, such as maintenance of stemness, regulation of hypoxia, epithelialto-mesenchymal transition (EMT), and escape of immune surveillance [7, 10-13]. Our group and Shi's group have proved inhibition of KDM1A can convert tumors from "cold" to "hot" via regulating the tumor immunogenicity $[7,13]$ and suppose KDM1A as a target to enhance the efficacy of immunotherapy on poor immunogenic cancers. However, the role of KDM1A in other cancers remains unknown. To date, there is no comprehensive study on the prognostic significance of KDM1A in pan-cancer.

In this study, we performed pan-cancer analysis by using the TCGA project and GTEx databases to systematically characterize the role of KDM1A across various cancer types. We conducted analyses of a set of elements, such as RNA level, protein level, survival curve, DNA methylation, genetic alteration, post-translation modification, microenvironment score, and relevant cellular pathway, to explore the potential mechanism of KDM1A in the pathogenesis or clinical prognosis of different cancers [14]. The current evidence suggested that KDM1A plays different roles in diverse cancers, and the underlying molecular mechanisms that occur in several cancers merit further investigation.

\section{Materials and Methods}

2.1. Gene Expression Analysis. The TIMER2.0 database was used to detect the expression difference of KDM1A using TCGA pan-cancer data [15]. GEPIA2 was used to draw the expression level of KDM1A in tumors and compare with related normal tissue from Genotype Tissue expression (GTEx) database, setting as $|\log 2 \mathrm{FC}|=1, p$ value $=0.05$, and "Match TCGA normal and GTEx data" [16]. Additionally, GEPIA2 was used to obtain violin plots of the KDM1A expression according to the tumor pathological stages.

To evaluate differences in KDM1A expression at the protein level, Clinical Proteomic Tumor Analysis Consortium (CPTAC) was analyzed using the UALCAN portal [17]. The expression levels of the total protein and phosphorylated protein of KDM1A (NP_001350583.1, NP_055828.2) were analyzed by comparison of the primary tumor and normal tissues.

The Oncomine database (https://www.oncomine.org/ resource/main.html) was also applied to obtain the different expressional levels of KDM1A between cancer and normal tissues by entering the word "KDM1A" and setting the threshold of $p$ value $=0.05$, fold change $=2$, and gene rank in top $10 \%$.

2.2. Survival Analysis. We used the "Survival AnalysisSurvival Map" module of GEPIA2 to obtain the effect of KDM1A expression on overall survival (OS) and disease-free survival (DFS) of various cancers based on TCGA. The highand low-expression cohorts were cut with the ratio of $50: 50$. The hypothesis test used a log-rank test. The "Survival Analysis" module was used to analyze the survival curve of each cancer type. The hazards ratio (HR) based on Cox $\mathrm{PH}$ model was calculated, and the $95 \%$ confidence interval (CI) as the dotted line is added in the figures.

2.3. Genetic Alteration Analysis. The cBioPortal (http:// cbioportal.org) website was used to rank the genetic variation of KDM1A via the "Cancer Types Summary" module, including the gene alteration frequency, mutation type, and copy number alteration (CNA) [18]. The mutated site of KDM1A was shown in the schematic diagram of the protein structure via the "Mutations" module. PyMol software was used to label mutation sites of KDM1A. The "Comparison" module was used to obtain the Kaplan-Meier curves of the OS, DFS, progression-free survival (PFS), and disease-specific survival (DSS) for various cancer types according to the $K D M 1 A$ genetic alteration. The log-rank $p$ value was shown. The mutation of KDM1A in the different subtypes of breast cancer was analyzed with the Breast Invasive Carcinoma data set (TCGA, Pan-Cancer Atlas) through cBioPortal.

2.4. DNA Methylation Analysis. MethSurv is an interactive and user-friendly web portal providing univariable and multivariable survival analysis based on DNA methylation biomarkers using TCGA (The Cancer Genome Atlas) data [19]. We evaluated survival data of all cancer types using DNA methylation of KDM1A as conditions, selecting the curves with $p$ value $<0.05$ to exhibit. Moreover, MEXPRESS was applied to visualize DNA methylation, expression, and clinical data [20].

2.5. Immune Infiltration Analysis. The TIMER2.0 database was used to analyze associations between KDM1A and tumor stromal cells, tumor-infiltrating immune cells, including cancer-associated fibroblasts, $\mathrm{CD}^{+} \mathrm{T}$ cells, $\mathrm{CD} 4^{+}$ T cells, Tregs, B cells, macrophages, myeloid-derived suppressor cells (MDSCs), neutrophils, and dendritic cells. The EPIC, MCPCOUNTER, TIDE, TIMER, CIBERSORT, CIBERSORT-ABS, QUANTISEQ, and XCELL algorithms were applied for estimations. The purity-adjusted Spearman's rank correlation test was used to obtain the $p$ values and partial correlation (cor) values, and then heatmaps and corresponding scatter plots were generated.

2.6. KDM1A-Related Gene Enrichment Analysis. The STRING database was used to acquire KDM1A-binding proteins [21]. We searched "KDM1A" in "Homo sapiens" and set main parameters, including Network type as "full STRING", the meaning of network edges as "evidence", active interaction sources as "experiments", the minimum required interaction score as "low confidence (0.150)", and the max number of interactors to show as "custom value; max interactors (100)" in the 1st shell. Finally, the available experiment-determined KDM1A-binding proteins were obtained as Set 1.

GEPIA2 was used to obtain 100 top KDM1A-correlated genes based on TCGA and GTEx databases as Set 2 via the "Similar Gene Detection" module. The "Correlation 
Analysis" module was used to execute a pairwise gene Pearson correlation analysis based on expression data. The dot plots showed $\log _{2}$ (TPM) with $p$ values and the correlation coefficient $(R)$. TIMER2.0 was applied to generate the heatmap to demonstrate the relationship between KDM1A and selected genes via the "Gene_Corr" module in the "Exploration" part.

Venny2.1.0 (https://bioinfogp.cnb.csic.es/tools/venny/ index.html) was applied to conduct an intersection analysis of Set 1 and Set 2 for the common genes. Moreover, we combined Set 1 and Set 2 to perform KEGG (Kyoto Encyclopedia of Genes and Genomes) pathway analysis and GO (Gene Ontology) enrichment analysis. We used the "clusterProfiler" R package to conduct KEGG enrichment analysis and GO enrichment analysis [22]. The enriched pathways were visualized with the bubble plots. GO enrichment analyses were visualized as bubble plots and cnetplots. The $R$ language software [R-3.6.3, 64-bit] (https:// www.r-project.org/) was used in this analysis. Two-tailed $p<0.05$ was considered statistically significant.

\section{Results}

3.1. KDM1A Gene Differentially Expressed between Normal and Tumor Tissues. TIMER2.0 was used to detect the differential expression of KDM1A between tumor and corresponding normal tissues from TCGA. The results showed that KDM1A was highly expressed in 15 cancer types compared with normal samples, including bladder urothelial carcinoma (BLCA), breast invasive carcinoma (BRCA), cholangiocarcinoma (CHOL), colon adenocarcinoma (COAD), esophageal carcinoma (ESCA), head and neck squamous cell carcinoma (HNSC), liver hepatocellular carcinoma (LIHC), lung adenocarcinoma (LUAD), lung squamous cell carcinoma (LUSC), prostate adenocarcinoma (PRAD), rectum adenocarcinoma (READ), stomach adenocarcinoma (STAD), uterine corpus endometrial carcinoma (UCEC), cervical squamous cell carcinoma and endocervical adenocarcinoma (CESC), and glioblastoma multiforme (GBM), and was lowly expressed only in kidney chromophobe $(\mathrm{KICH})$, kidney renal clear cell carcinoma (KIRC), and kidney renal papillary cell carcinoma (KIRP) (Figure 1(a)).

As the corresponding normal tissues of 10 cancer types are unavailable in the TCGA database, we used the expression data of normal tissues from the GTEx database to compare with TCGA data (Figures 1(b) and S1(a)). It was shown that the KDM1A gene was highly expressed in tumor samples of lymphoid neoplasm diffuse large B-cell lymphoma (DLBC) and thymoma (THYM) and was lower in acute myeloid leukemia (LAML) compared with normal tissues $(p<0.05)$. Moreover, 7 cancers showed no significant difference in the expression of KDM1A compared with normal tissues (Figure S1(a)).

We further explored the transcription levels of KDM1A in cancer using the Oncomine database (Figure 1(c)). Relative to normal tissues, KDM1A in bladder cancer, colorectal cancer, kidney cancer, leukemia, and lung cancer was overexpressed, while it was downregulated in brain and CNS cancer and breast cancer, which made the potential function as either oncogenic or antitumor activities based on the cancer types. Part of Oncomine data was inconsistent with the analysis of TCGA data, perhaps caused by different sample sources and different tumor classifications. Hence, detailed analyses of KDM1A are considered for further analysis.

To evaluate the protein level of KDM1A, CPTAC was utilized to analyze the TCGA data. As shown in Figure 1(d), the total protein level of KDM1A was higher in breast cancer, uterine corpus endometrial carcinoma (UCEC), colon cancer, ovarian cancer, lung adenocarcinoma $(p<0.001)$, and clear cell RCC $(p<0.05)$ compared with normal tissues.

Moreover, we applied GEPIA2 to investigate the correlation of KDM1A with the pathological stages. KDM1A expression was a positive correlation with pathological stages in 4 cancers, including LIHC, HNSC, SKCM, and OV, but not others (Figures 1(e) and Figure S1(b)).

3.2. Survival Analysis of KDM1A. To investigate the association of KDM1A expression with prognosis, survival association analysis was performed via GEPIA2 based on the expression level of KDM1A. The cancer cases were dichotomized into high and low groups according to KDM1A expression. As shown in Figures 1(f) and S2, the high-expression group was linked to poor OS (overall survival) for cases of ACC $(p=0.0014)$, LIHC $(p=0.0053)$, and SARC $(p=0.011)$, and the contrary result was shown for cases of COAD $(p=0.023)$ and KIRC $(p=0.025)$. Additionally, DFS (disease-free survival) was analyzed and showed that 4 cancer types with high KDM1A were positively related to poor prognosis, including ACC $(p=4.2 e-05)$, LIHC $(p=0.021), \mathrm{KICH}(p=0.026)$, and LGG $(p=0.017)$, and low KDM1A was associated with poor DFS for KIRC $(p=0.015)$.

The Kaplan-Meier plotter tool was also utilized to analyze the expression of the KDM1A gene concerning clinical prognosis. The result presented that the high expression of KDM1A was associated with better OS $(p=0.0068)$ but the reverse effect to RFS $(p=0.001)$ in patients with breast cancer (Figure S3(a)). In ovarian cancer, the high KDM1A group was related to poor OS $(p=0.043)$ and PFS $(p=0.02)$ (Figure S3(b)). The low expression of KDM1A in gastric cancer was associated with poor PPS $(p=0.0013)$ (Figure S3(c)). The upregulation of KDM1A was correlated with poor OS $(p=0.0031)$ in LUAD (Figure S3(d)). The downregulation of KDM1A was linked to poor PPS $(p=0.072)$ in LUSC (Figure S3(e)). Moreover, highly expressed KDM1A was coupled with poor OS, RFS, PFS, and DSS (all $p<0.001$ ) for the cases of liver cancer (Figure S3(f)). The summary of the differential association between KDM1A expression and the prognosis of different cancers is shown in Table 1, according to both methods of GEPIA2 and Kaplan-Meier plotter.

3.3. Genetic Alterations of KDM1A. We applied cBioPortal to observe the chromosomal abnormalities and mutation status of KDM1A in various cancers using the TCGA data. 


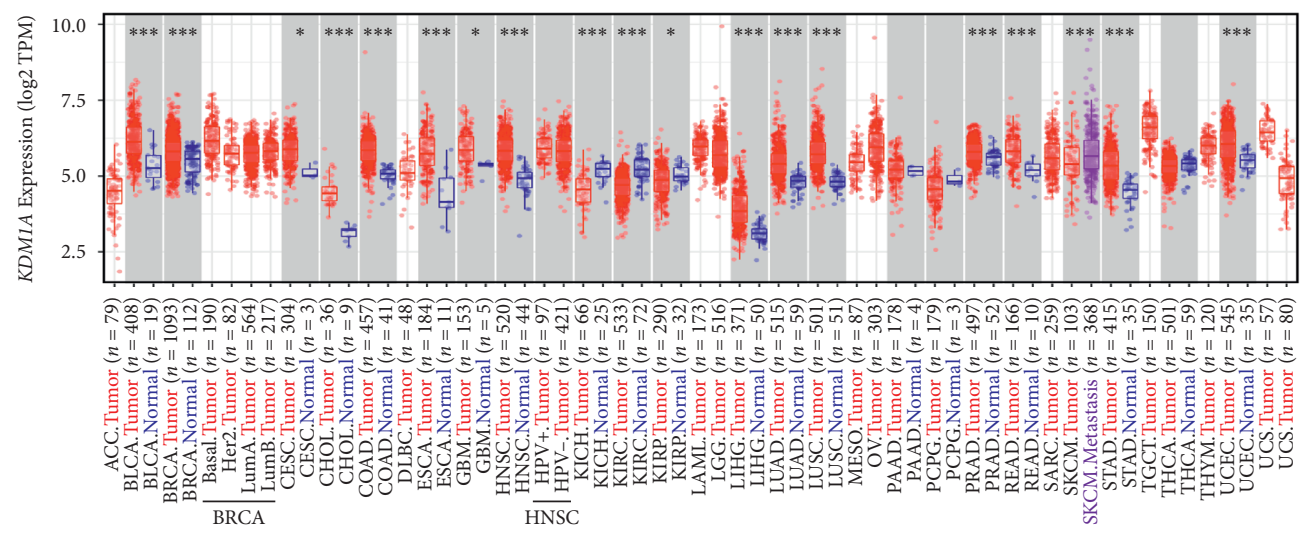

(a)
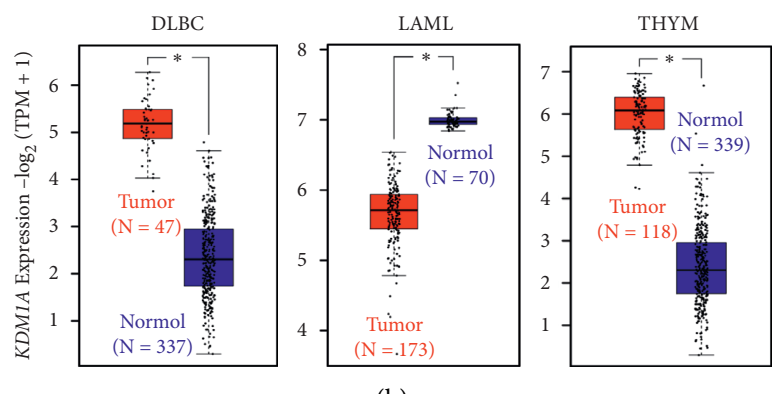

(b)

Disease Summary for KDM1A

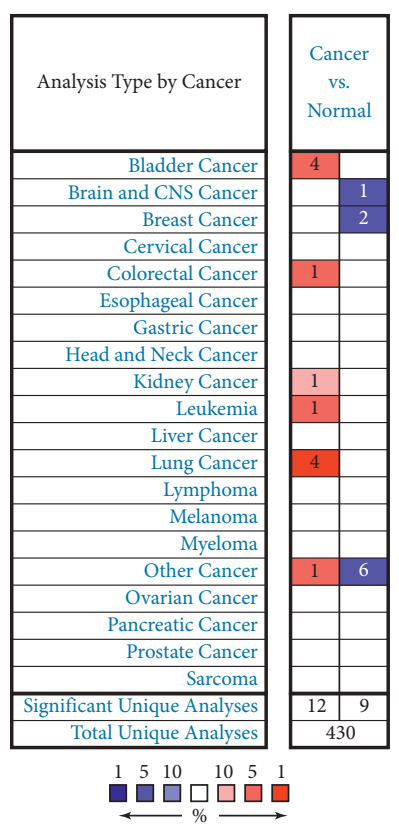

Gene rank percentile (\%)

(c)

FIgUre 1: Continued. 

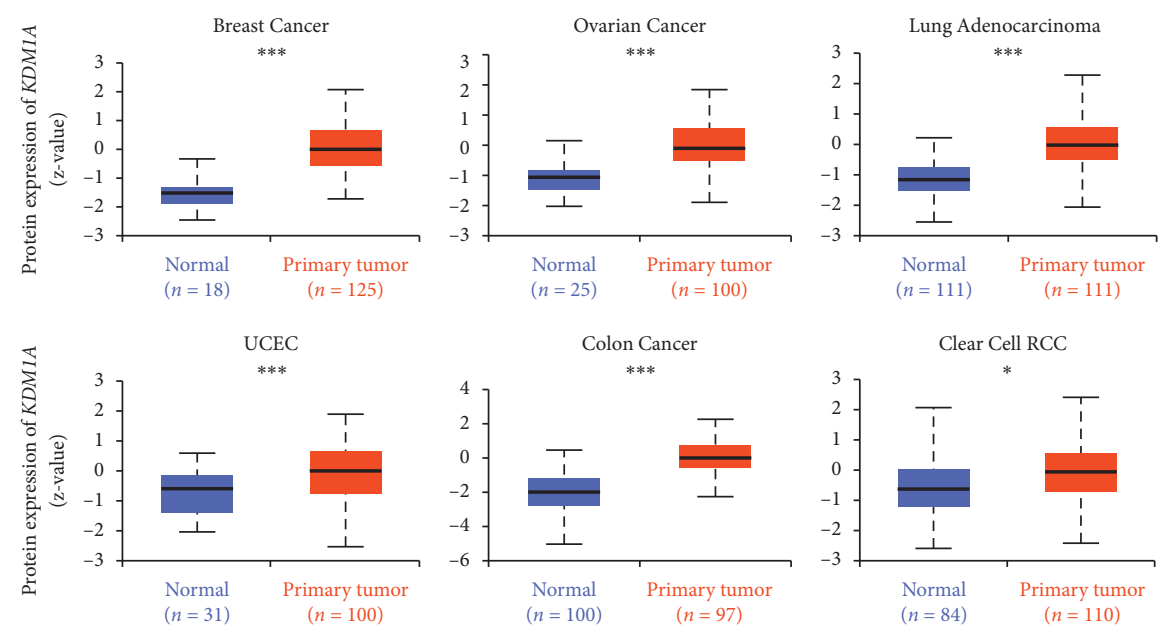

(d)
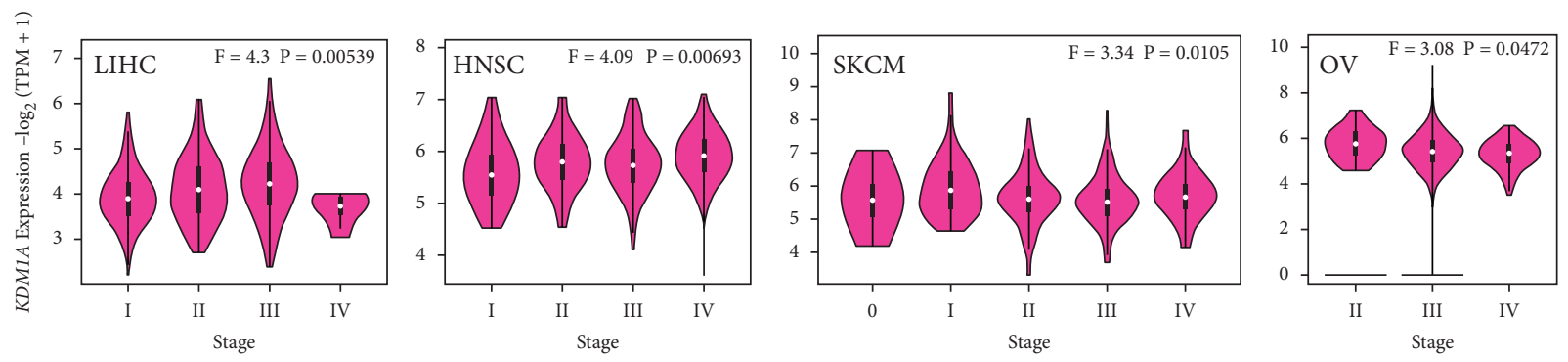

(e)

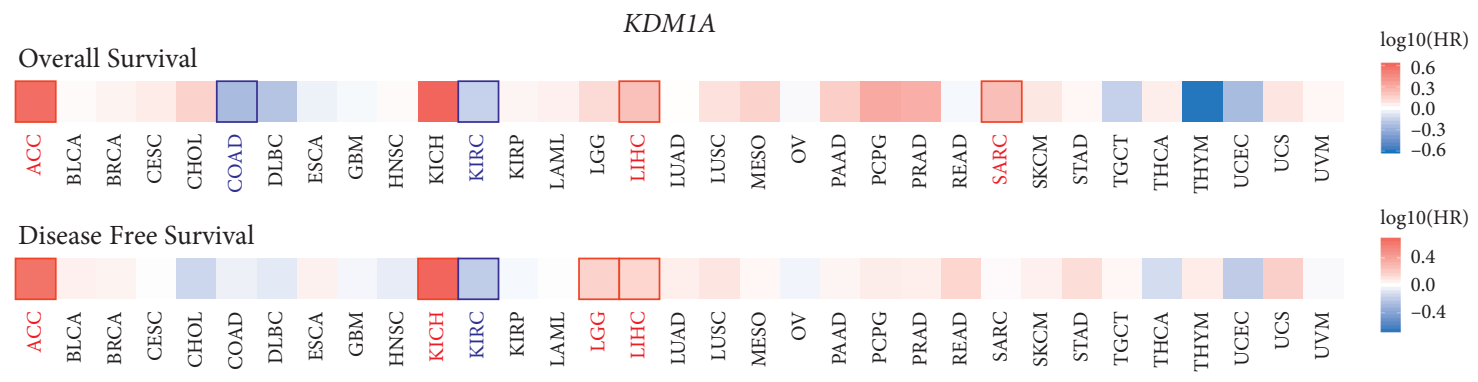

(f)

Figure 1: Analysis of the expressional level of KDM1A gene and survival prognosis of cancers. (a) TIMER2.0 was used to analyze the expressional level of the KDM1A in different cancers. (b) The box plot data were supplied for the type of DLBC, LAML, and THYM in the TCGA project, and the corresponding normal tissues of the GTEx database were included as controls. (c) Expressional levels of KDM1A in different types of tumors according to the Oncomine database. The plot indicated the numbers of datasets with statistically significant $(p<0.05)$ mRNA overexpression (red) or downexpression (blue) of KDM1A (different types of cancer vs. corresponding normal tissue). (d) The protein expressional levels of KDM1A were analyzed according to the CPTAC dataset. (e) The main pathological stages of KDM1A expression levels in LIHC, HNSC, SKCM, and OV based on the TCGA data. (f) Survival prognosis of cancers including overall survival and disease-free survival. ${ }^{*} p<0.05 ;{ }^{* *} p<0.01$; ${ }^{* * *} p<0.001$.

As illustrated in Figure 2(a), uterine cancer owned the highest alteration frequency of KDM1A $(>4 \%)$ with mutation frequency as the main proportion. It is worth mentioning that deep deletion of KDM1A accounted for all cases of genetic alteration in $\mathrm{CHOL}$, pheochromocytoma and paraganglioma (PCPG), DLBC, mesothelioma (MESO), THYM, TGCT, and KIRC. Meanwhile, all cases of KDM1A alteration were the amplification of copy number in UCS and SARC. We further present the sites and types of KDM1A mutation and related case numbers in Figure 2(b). The missense mutation was the highest among genomic alterations, which include the alterations of $\mathrm{R} 321 \mathrm{C} / \mathrm{H}$, $\mathrm{E} 477 \mathrm{~K}$, and $\mathrm{R} 591 * / \mathrm{L}$ in the amino oxidase domain, including 3 cases each and involving SKCM, UCEC, BLCA, LUSC, and CESC (Figure 2(b)). As shown in the 3D structure of KDM1A protein, R321 and R591 located at the region of the KDM1A catalytic pocket, while E477 stood at the binding region of KDM1A with the nucleosome and coeffector (Figure 2(c)). Moreover, we present the alteration sites of all TCGA cancer types in Table S1. Furthermore, we investigated the association between the clinical survival of cases and KDM1A mutations with various cancers. As 
TABLE 1: The summary of analysis on KDM1A expression and prognosis in different tumors of TCGA

\begin{tabular}{|c|c|c|c|c|c|c|}
\hline & Tumor type & $\begin{array}{l}\text { mRNA } \\
\text { expression }\end{array}$ & $\begin{array}{l}\text { Protein } \\
\text { expression }\end{array}$ & Stage level & $\begin{array}{l}\text { Poor prognosis } \\
\text { of OS }\end{array}$ & $\begin{array}{l}\text { Poor prognosis of } \\
\text { DFS }\end{array}$ \\
\hline ACC & Adrenocortical carcinoma & ns & NA & ns & Positive** $^{* *}$ & Positive P $^{* * *}$ \\
\hline BLCA & Bladder urothelial carcinoma & High*** $^{* *}$ & NA & ns & ns & ns \\
\hline BRCA & Breast invasive carcinoma & High $^{* * *}$ & High $^{* * *}$ & ns & Negative** $^{* *}$ & ns \\
\hline CESC & $\begin{array}{l}\text { Cervical squamous cell carcinoma and endocervical } \\
\text { adenocarcinoma }\end{array}$ & High* & NA & ns & ns & ns \\
\hline $\mathrm{CHOL}$ & Cholangiocarcinoma & High*** $^{* *}$ & NA & ns & ns & ns \\
\hline COAD & Colon adenocarcinoma & High*** $^{* *}$ & High $^{* * *}$ & ns & Negative* $^{*}$ & ns \\
\hline DLBC & Lymphoid neoplasm diffuse large B-cell lymphoma & High $^{* *}$ & NA & ns & ns & ns \\
\hline ESCA & Esophageal carcinoma & High $^{* * *}$ & $\mathrm{NA}$ & ns & ns & ns \\
\hline GBM & Glioblastoma multiforme & High* & $\mathrm{NA}$ & NA & ns & ns \\
\hline HNSC & Head and neck squamous cell carcinoma & High*** $^{* *}$ & NA & $\mathbf{F}=4.09^{* *}$ & ns & ns \\
\hline $\mathrm{KICH}$ & Kidney chromophobe & Low $^{* * *}$ & NA & ns & ns & Positive* \\
\hline KIRC & Kidney renal clear cell carcinoma & Low $^{* * *}$ & High* & ns & Negative $^{*}$ & Negative* \\
\hline KIRP & Kidney renal papillary cell carcinoma & Low ${ }^{*}$ & NA & ns & ns & ns \\
\hline LAML & Acute myeloid leukemia & Low $^{* *}$ & NA & NA & ns & ns \\
\hline LGG & Brain lower grade glioma & ns & NA & NA & ns & Positive* \\
\hline LIHC & Liver hepatocellular carcinoma & High $^{* * *}$ & NA & $\mathbf{F}=4.3^{* *}$ & Positive $^{* *}$ & Positive* \\
\hline LUAD & Lung adenocarcinoma & High $^{* * *}$ & High $^{* * *}$ & ns & Positive** & ns \\
\hline LUSC & Lung squamous cell carcinoma & High*** $^{*}$ & NA & ns & ns & ns \\
\hline MESO & Mesothelioma & NA & NA & NA & ns & ns \\
\hline $\mathrm{OV}$ & Ovarian serous cystadenocarcinoma & ns & High $^{* * *}$ & $\mathrm{~F}=3.08^{*}$ & Positive* $^{*}$ & ns \\
\hline PAAD & Pancreatic adenocarcinoma & ns & NA & ns & ns & ns \\
\hline PCPG & Pheochromocytoma and paraganglioma & ns & NA & NA & ns & ns \\
\hline PRAD & Prostate adenocarcinoma & High $^{* * *}$ & $\mathrm{NA}$ & NA & ns & ns \\
\hline READ & Rectum adenocarcinoma & High $^{* * *}$ & NA & ns & ns & ns \\
\hline SARC & Sarcoma & ns & $\mathrm{NA}$ & NA & Positive* & ns \\
\hline SKCM & Skin cutaneous melanoma & ns & NA & $\mathbf{F}=3.34^{*}$ & ns & ns \\
\hline STAD & Stomach adenocarcinoma & High $^{* * *}$ & NA & ns & ns & ns \\
\hline TGCT & Testicular germ cell tumors & ns & NA & ns & ns & ns \\
\hline THCA & Thyroid carcinoma & ns & NA & ns & ns & ns \\
\hline THYM & Thymoma & High $^{* *}$ & NA & NA & ns & ns \\
\hline UCEC & Uterine corpus endometrial carcinoma & High*** $^{* *}$ & High $^{* * *}$ & ns & ns & ns \\
\hline UCS & Uterine carcinosarcoma & ns & NA & ns & ns & ns \\
\hline UVM & Uveal melanoma & NA & NA & NA & ns & ns \\
\hline
\end{tabular}

OS, overall survival; DFS, disease-free survival; NA, not available; ns, no significance; $* p<0.05 ; * * p<0.01 ; * * * p<0.001$.

shown in Figure 2(d), breast invasive carcinoma cases with KDM1A alteration indicated poor OS $(p=0.0391)$, DSS $(p=2.493 e-03)$, PFS $(p=0.0284)$ survival, but not DFS ( $p=0.230$ ), compared with cases without KDM1 A mutation. Subsequently, we surveyed the association of breast cancer subtype and the KDM1A alteration and found 5 of 7 cases with $K D M 1 A$ alteration were luminal A type of breast cancer (Figure 2(e)).

3.4. DNA Methylation Analysis of KDM1A. To investigate the DNA methylation of $K D M 1 A$, we explore the data of KDM1A DNA methylation of different cancer types in the TCGA project. As displayed in Table 2, the methylation level of the KDM1A promoter region was negatively correlated with gene expression in BRCA, KIRC, MESO, READ, SKCM, and UCEC and positively correlated in HNSC and LUSC. In LGG, the methylation level at cg22683154 was negatively correlated with gene expression, whereas methylation at $\operatorname{cg} 06958034$ was a positive correlation with gene expression. Moreover, the level of methylation was a negative correlation with gene expression based on multiple probes of the nonpromoter region $(p<0.05)$. We further analyzed the potential correlation of KDM1A DNA methylation with the prognosis of different cancers via MethSurv and MEXPRESS approach, and the results showed that hypermethylation of KDM1A is positively correlated with good prognosis in most tumors (Figures 3 and S4).

3.5. Phosphorylation Levels of KDM1A Protein. To compare phosphorylation levels of KDM1A between normal tissues and primary tumor tissues, six cancer types (breast cancer, ovarian cancer, clear cell RCC, LUAD, UCEC, and COAD) were analyzed via the CPTAC dataset. The phosphorylation levels of KDM1A protein in different tumors are framed in Table S2. As shown in Figure 4(a), the phosphorylation sites of KDM1A with significant differences $(p<0.05)$ were summarized, and the most frequent phosphorylation sites were located at the $\mathrm{N}$-terminal. Compared with normal tissues, the phosphorylation levels of different sites were upregulated in breast cancer, colon cancer, UCEC, and LUAD and downregulated in clear cell RCC, ovarian cancer, and colon cancer. Interestingly, different phosphorylation 


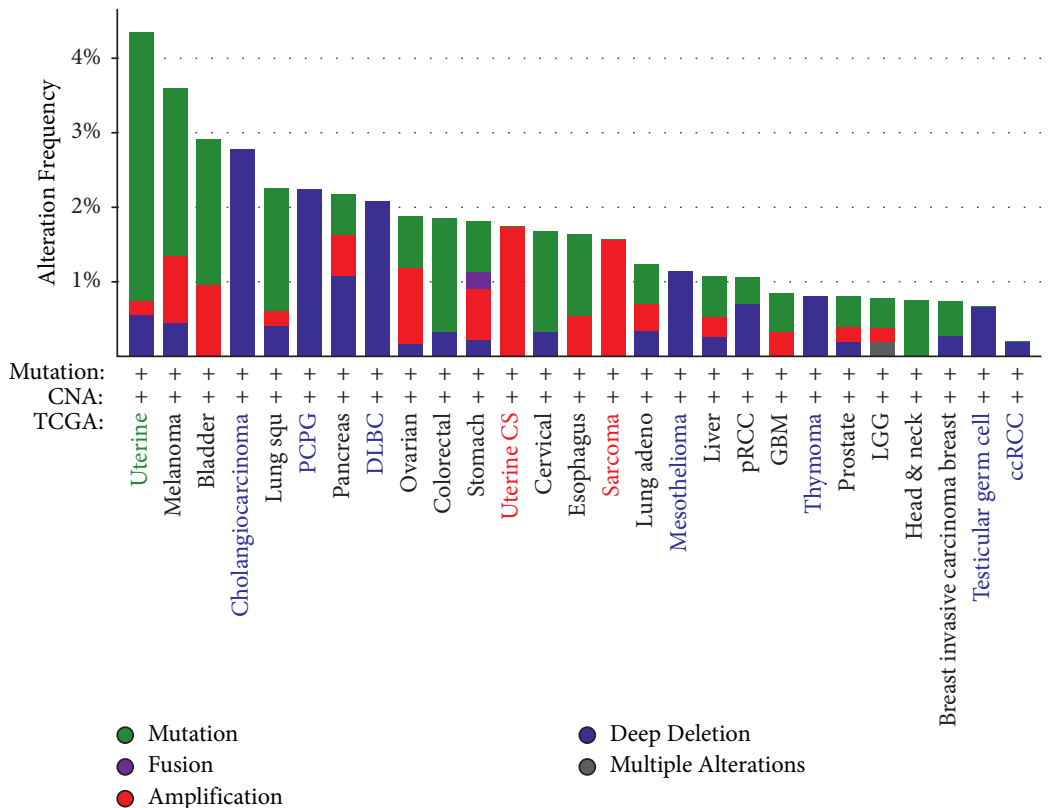

(a)

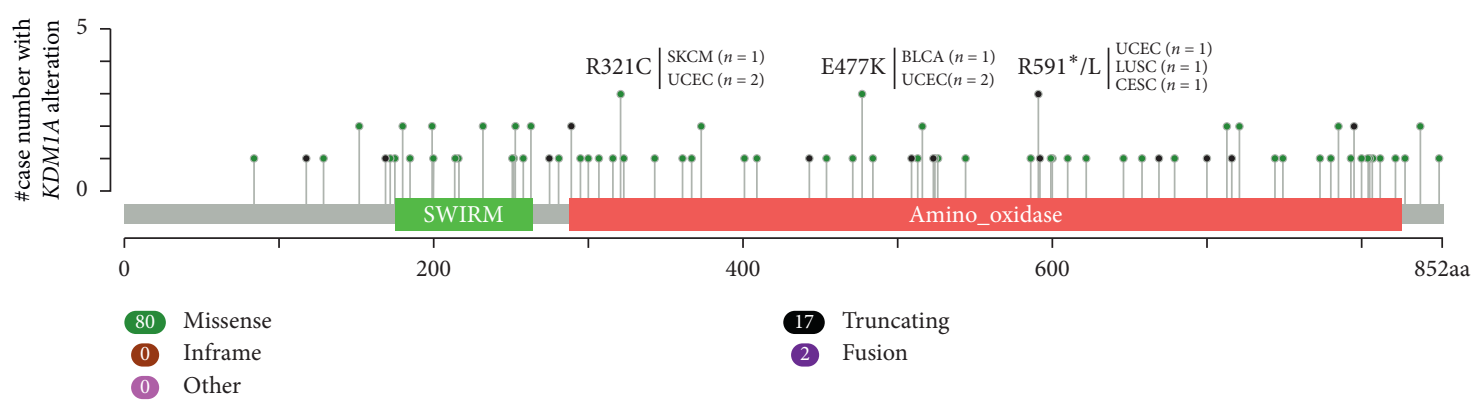

(b)

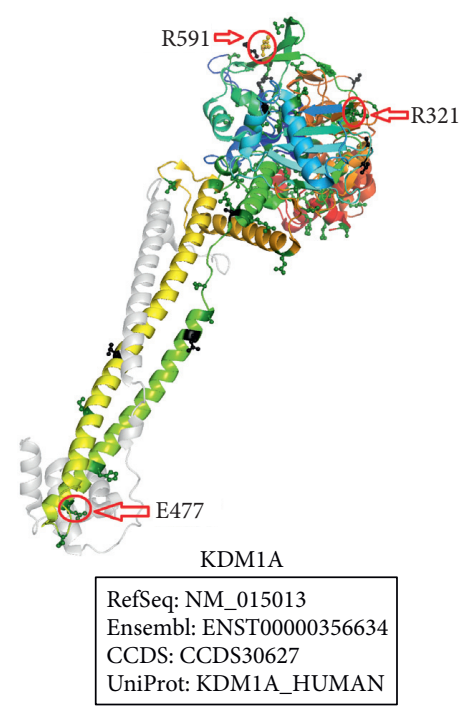

(c)

Figure 2: Continued. 

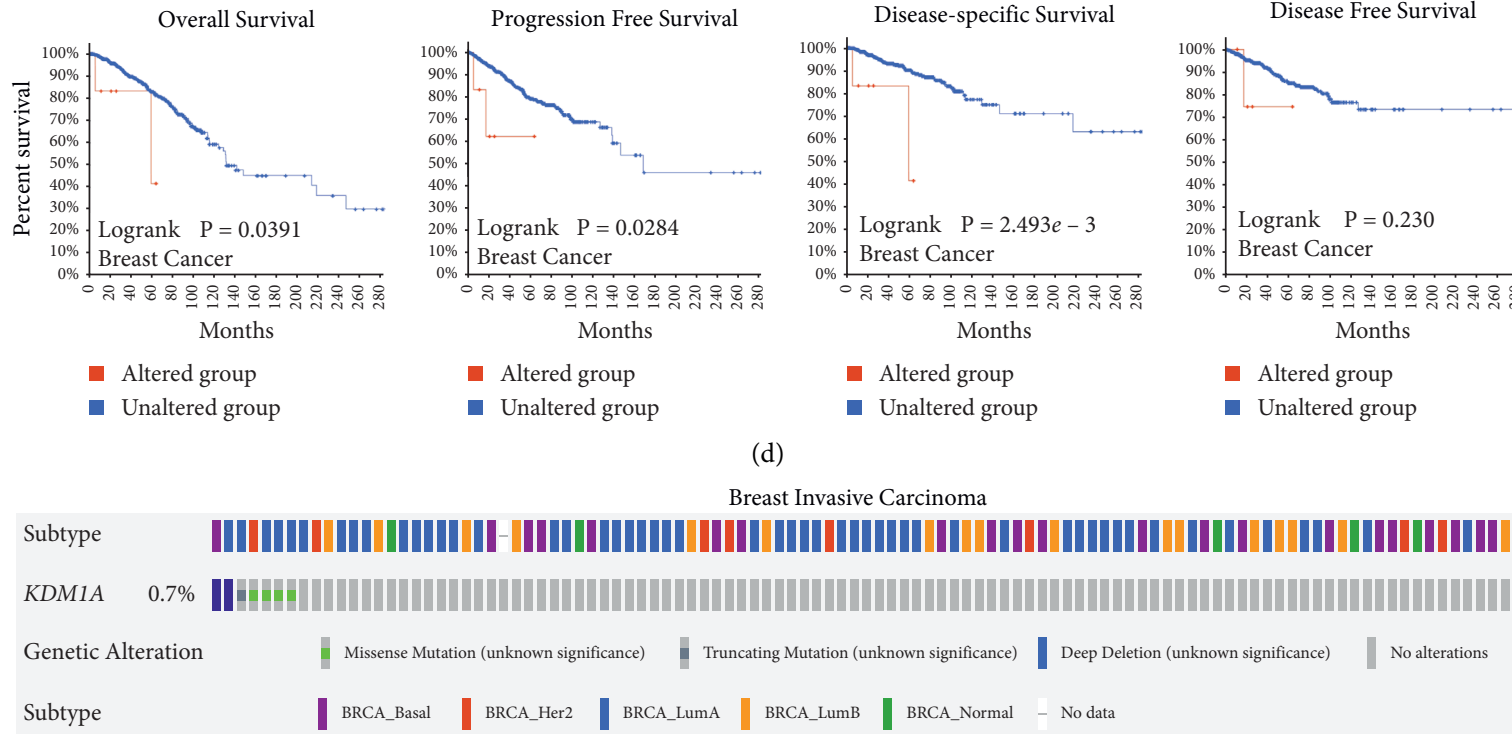

(e)

FIGURE 2: KDM1A mutations in different tumors according to the TCGA data. (a) The alteration frequency of KDM1A with mutation type using the cBioPortal tool. (b) KDM1A mutation site and corresponding diseases of the highest number of cases are displayed. (c) The top three mutation sites including R321C/H, E477K, and R591 * /L showed in the 3D structure of KDM1A. (d) Mutation status of KDM1A was relevant to the OS, PFS, DSS, and DFS of breast cancer analyzed by the cBioPortal tool. (e) Breast cancer samples with KDM1A mutation were identified from the TCGA Invasive Breast Carcinoma data set.

TABLE 2: Relationship between KDM1A DNA methylation and gene expression.

\begin{tabular}{lcccc}
\hline Cancer & Name & pearson_r & $p$ value & $\begin{array}{c}\text { The promoter } \\
\text { probe }\end{array}$ \\
\hline \multirow{2}{*}{ BRCA } & cg04886391 & -0.0870 & 0.010281191 & Yes \\
& cg25977026 & -0.5540 & $5.95276 E-40$ & No \\
CESC & cg25977026 & -0.4934 & $2.31587 E-18$ & No \\
& cg23271558 & -0.1294 & 0.022886159 & No \\
HNSC & cg12578844 & 0.1234 & 0.004768733 & Yes \\
& cg25977026 & -0.4335 & $1.0084 E-21$ & No \\
KIRC & cg22683154 & -0.2429 & $5.65216 E-06$ & Yes \\
& cg03967533 & -0.1554 & 0.003970689 & No \\
LGG & cg22683154 & -0.0875 & 0.045136678 & Yes \\
LIHC & cg06958034 & 0.2343 & $6.17708 E-08$ & Yes \\
LUAD & cg25977026 & -0.5302 & $3.75586 E-25$ & No \\
LUSC & cg26662347 & -0.5043 & $1.48899 E-25$ & No \\
MESO & cg07118078 & -0.1615 & 0.001653858 & Yes \\
READ & cg22683154 & -0.2815 & 0.003163089 & Yes \\
SARC & cg25977026 & -0.4172 & $4.05178 E-12$ & No \\
SKCM & cg22683154 & -0.1241 & 0.007028282 & Yes \\
STAD & cg23271558 & -0.2353 & $1.25499 E-05$ & No \\
UCEC & cg04886391 & -0.1419 & 0.002202533 & Yes
\end{tabular}

sites showed converse regulation in colon cancer. The phosphorylation levels of S69 and S131 were upregulated and the level of S166 was downregulated in colon cancer. We further found that the S131 locus exhibits a higher phosphorylation level in breast cancer, colon cancer, UCEC, and LUAD compared with normal tissues but lower in renal clear cell carcinoma and the S131 locus can undergo double phosphorylation in conjunction with other phosphorylation sites (Figures 4(b), 4(c), and S5). Furthermore, we also utilized PhosphoNET to analyze the phosphorylation of KDM1A in the CPTAC database (Table S3). One publication experimentally revealed the biological significance of phosphorylation of LSD1 at S131 and S137 mediated by CK2, which benefited cell proliferation and survival after DNA damage [23]. This discovery indicates the significance of further experimental exploration for the role of KDM1A phosphorylation in tumorigenesis.

3.6. Relationship between KDM1A Expression and Tumor Microenvironment. Various algorithms in TIMER2.0 were applied to measure the potential correlation between KDM1A and cancer-associated fibroblast (CAF) and immune cells in diverse cancer types. Through multiple analyses, we observed a statistically positive correlation between KDM1A expression and CAF in most cancer types, but a negative correlation in THYM (Figure 5(a)). As for myeloidderived suppressor cells (MDSCs), it can be learned from the TIDE algorithm that MDSCs were positively correlated with KDM1A expression (Figure 5(c)). In addition, we noticed a negative correlation of KDM1A expression with the infiltration of $\mathrm{CD}^{8+} \mathrm{T}$ cells in TGCT, LGG, KIRP, KIRC, and HNSC-HPV+ based on most algorithms (Figure S6). The scatter plots are shown in Figures 5(b) and S6(b). For instance, the KDM1A level in CESC was positively associated with CAF (Figure 5, cor $=0.362, p=5.20 e-10$ ) depending on the EPIC algorithm. The correlation between the other tumor-infiltrating immune cells and KDM1A expression is shown in Figures S7 and S8. Interestingly, in most cancer types, KDM1A was negatively correlated with $\mathrm{CD}^{4+} \mathrm{Th} 1$ cells 

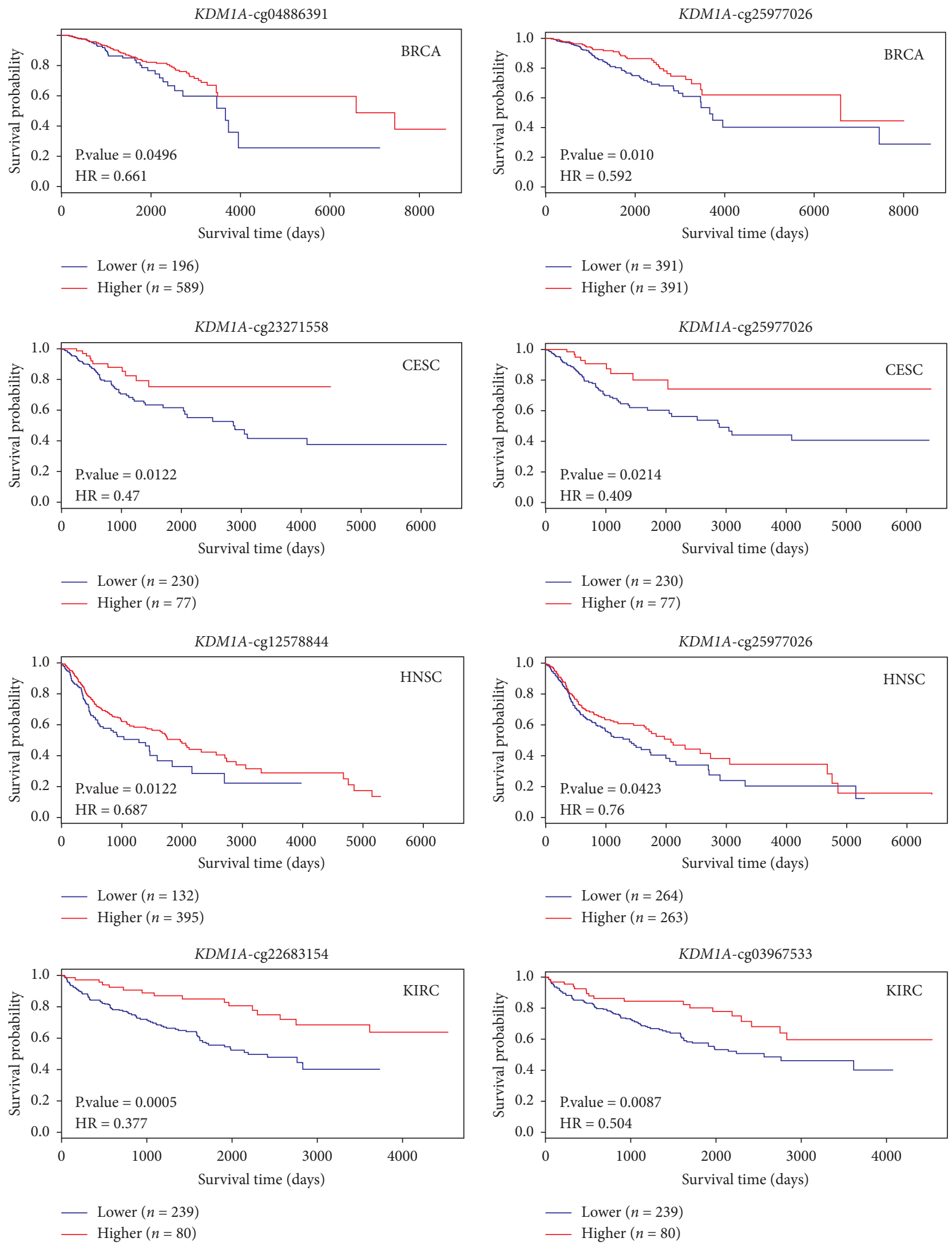

(a)

Figure 3: Continued. 

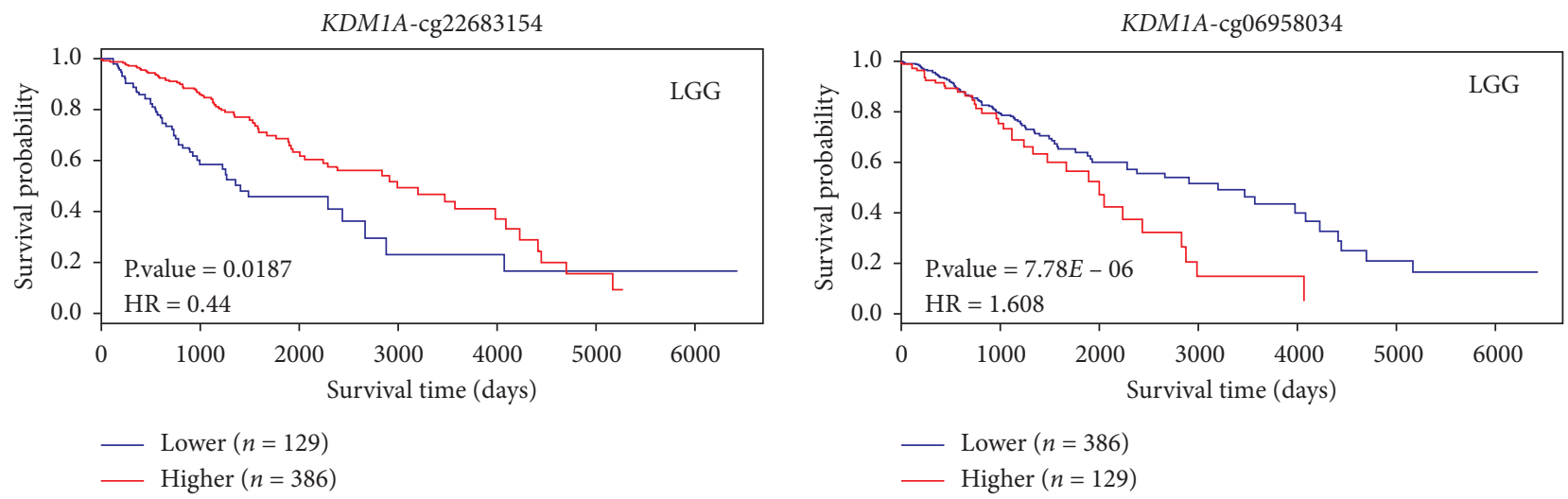

(b)

FIgURe 3: Correlation between DNA methylation of KDM1A and survival prognosis in TCGA tumors using MethSurv. The $p$ value $(<0.05)$ and the hazard ratio (HR) are displayed.

and positively correlated with $\mathrm{CD}^{4+} \mathrm{Th} 2$ cells (Figure S7(a)). In addition, there was a positive correlation between Tregs and KDM1A expression in LIHC and LGG, but a negative correlation in TGCT (Figure S7(b)). B-cell infiltration was negatively correlated with KDM1A expression in STAD, READ, and HNSC (Figure S7(c)). Moreover, neutrophil infiltration was positively correlated with KDM1A expression in multiple tumors from various algorithms (Figure S8(c)), whereas other myeloid cells, such as macrophages and dendritic cells, showed no obvious correlations with KDM1A in cancer types via different algorithms (Figures S8(a) and S8(b)).

3.7. Enrichment Analysis of KDM1A-Related Genes. To study the molecular significance of KDM1A in tumorigenesis and development, we screened out the KDM1A-binding proteins and expression-correlated genes for downstream analyses. We generated Set 1 including 100 KDM1A-binding proteins stood by experimental evidence via the STRING database. The protein-protein interaction networks of these proteins excluding histone-associated proteins are shown in Figure 6(a). GEPIA2 was applied to analyze all expression data of TCGA and yield Set 2 including the top 100 genes correlating with KDM1A expression. The expression of top 6 genes in Set 2 were shown to maintain positive correlation with KDM1A (Figure 6(b)), including DHX9 (DExH-box helicase 9) $(\mathrm{R}=0.58)$, SNRNP40 (small nuclear ribonucleoprotein U5 subunit 40) $(\mathrm{R}=0.59)$, HNRNPR (heterogeneous nuclear ribonucleoprotein $\mathrm{R})(\mathrm{R}=0.63)$, PPM1G (protein phosphatase, $\mathrm{Mg}^{2+} / \mathrm{Mn}^{2+}$-dependent $1 \mathrm{G}$ ) $(\mathrm{R}=0.51)$, HDAC2 (histone deacetylase 2) $(\mathrm{R}=0.54)$, and SMARCA4 (SWI/SNF related, matrix associated, actin-dependent regulator of chromatin, subfamily a, member 4) $(\mathrm{R}=0.48)$ (all $p<0.001)$. The positive correlations between KDM1A and the above six genes in different cancer types were displayed via a heatmap (Figure 6(c)). A Venn analysis of Set 1 and Set 2 generated two common genes, HDAC2 and SMARCA4 (Figure 6(d)).

Furthermore, we merged Set 1 and Set 2 to execute pathway and GO enrichment analyses. The KEGG-based pathway enrichment indicated that "spliceosome", "cell cycle", and "RNA transport" pathways were involved in the effect of KDM1A (Figure 6(e)). GO enrichment analysis indicated that KDM1A-related genes were enriched to the terms related to DNA and RNA, such as nucleosome binding, repressing transcription factor binding, chromatin DNA binding, RNA polymerase II transcription factor binding, RNA splicing, RNA localization, and others (Figures 7(a)-7(c)).

\section{Discussion}

Histone lysine methylation is an important covalent posttranslational modification (PTM) of chromatin. To date, two different families of histone demethylases (KDMs) have been identified as the flavin-dependent amine oxidase-containing and the Jumonji $\mathrm{C}$ (JmjC)-domain-containing enzymes that both use oxidative mechanisms to catalyze $\mathrm{N}$-methyl-lysine demethylation [24]. The first KDM (LSD1 or KDM1A) was identified by Shi's group in 2004 as a member of the FAD amine oxidase family [3]. KDM1A can demethylate $\mathrm{H} 3 \mathrm{~K} 4 \mathrm{me} 1 / 2$ and $\mathrm{H} 3 \mathrm{~K} 9 \mathrm{mel} / 2$ based on its interacting partners $[3,25]$. KDM1A demethylates $\mathrm{H} 3 \mathrm{~K} 4 \mathrm{me} 1 / 2$ and renders genes transcriptional repression via binding with CoREST (REST (RE1-silencing transcription factor) corepressor), CtBP (C-terminal-binding protein 1), and NuRD (nucleosome remodeling and deacetylase) complexes [26-29]. In addition, KDM1A interacting with androgen receptor (AR) or estrogen receptor (ER) induces transcriptional activation by demethylating H3K9me1/2 $[4,30,31]$. Furthermore, LSD1n, a neuron-specific isoform of LSD1 (KDM1A), was verified to specially target H4K20me2 for transcription activation of neuronal-regulated genes [5]. $\mathrm{KDM} 1 \mathrm{~B} / \mathrm{AOF} 1$, as another member of the KDM1s family, is a histone H3K4 demethylase [32]. KDM1B plays different roles in the regulation of proliferation, apoptosis, and stemness in several cancers, such as breast cancer, ovarian cancer, and pancreatic cancer [24, 33-35]. In this study, we used pan-cancer analysis to systematically characterize the roles of KDM1A. 


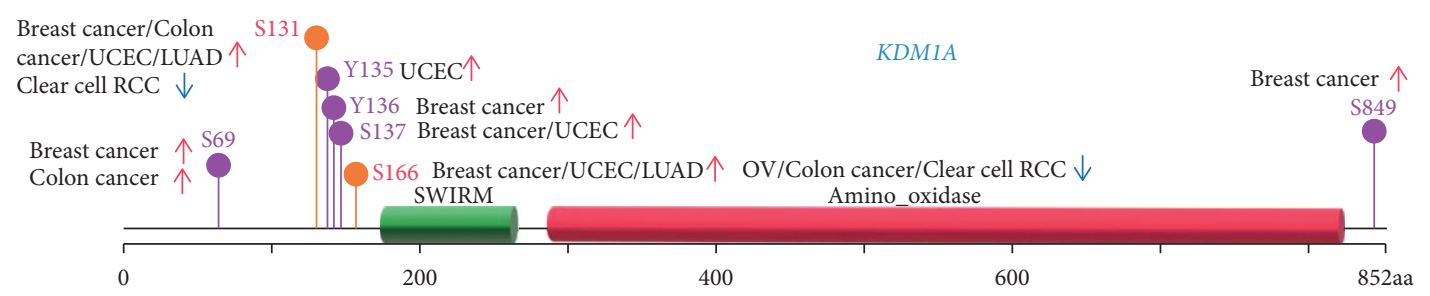

(a)
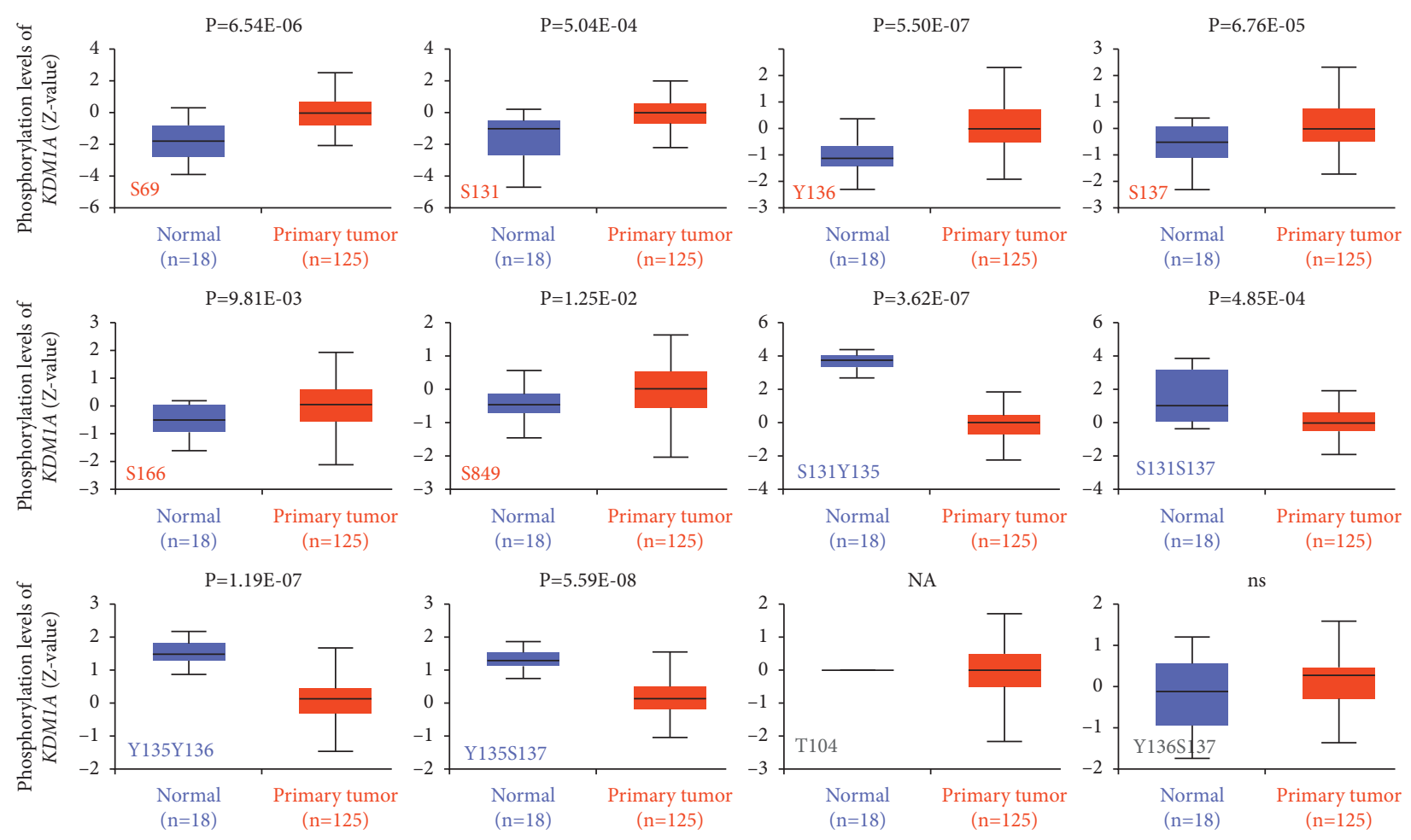

(b)

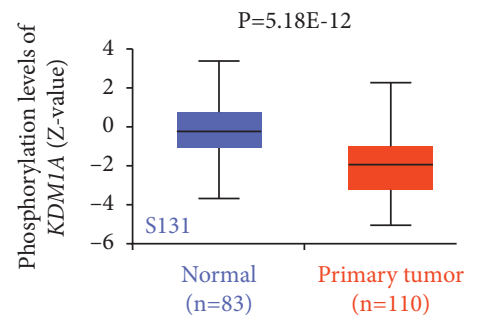

Clear cell RCC (NP_001350583.1)
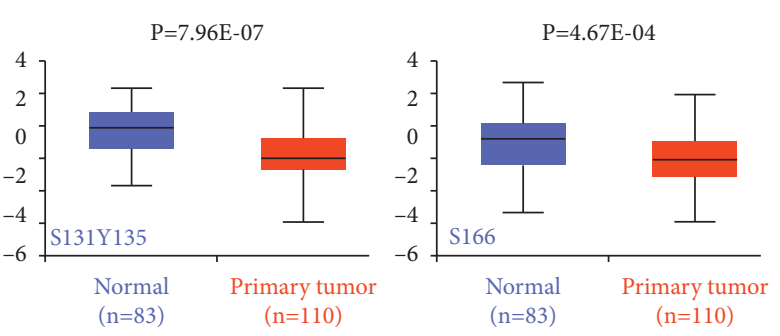

(c)

FIGURE 4: Analysis of phosphorylation levels of KDM1A in different cancers based on the CPTAC data set via the UALCAN. (a) Schematic diagram showed the phosphoprotein sites of KDM1A (NP_001350583.1) that were expressed at different levels in tumors compared with normal tissues. (b) and (c) Phosphorylation analysis of KDM1A protein in breast cancer and clear cell RCC, respectively.

Multiple studies showed that KDM1A expression is high in various cancers and plays an important role in different cancer-related processes. Considerable studies have highlighted the pivotal role of KDM1A in several cellular processes of normal and cancer cells such as stemness maintaining, differentiation [36, 37], cell migration, epithelial-to-mesenchymal transition [12], autophagy [38], senescence [39], neurodegenerative diseases [40], and metabolism [41]. However, a pan-cancer analysis of KDM1A was still urgently needed to reveal its relationship with cancer from the overall perspective. Thus, we comprehensively investigated the expression and efficacy of KDM1A on a total of 33 different cancer types in TCGA, GTEx, and CPTAC databases from the following aspects including gene expression, mutations, protein phosphorylation, DNA methylation, and tumor-infiltrating immune. 


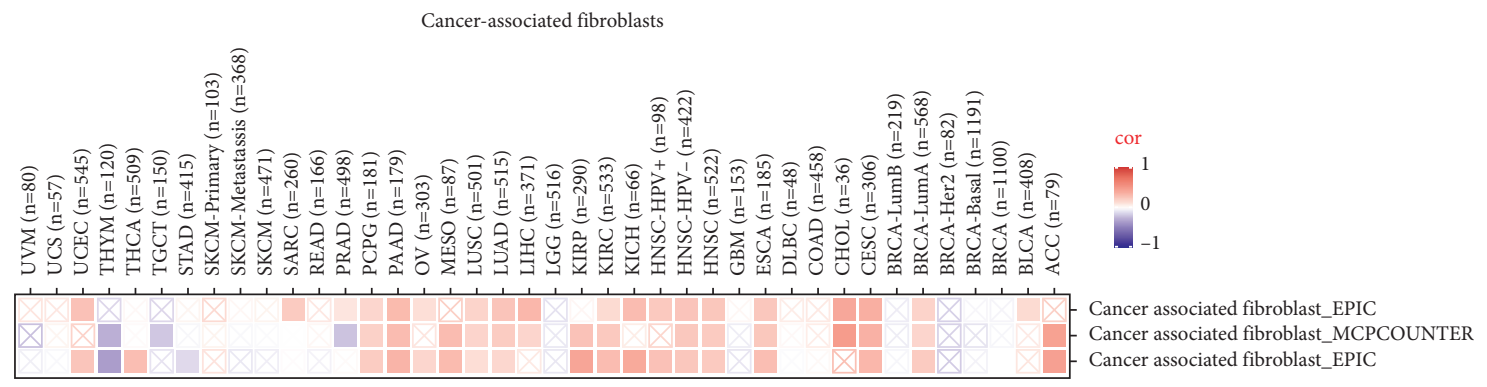

凶p>0.05

$\mathrm{p}<0.05$

(a)

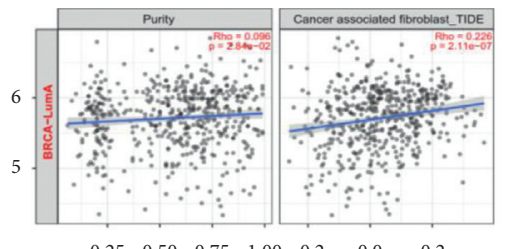

$\begin{array}{lllllll}0.25 & 0.50 & 0.75 & 1.00 & -0.2 & 0.0 & 0.2\end{array}$

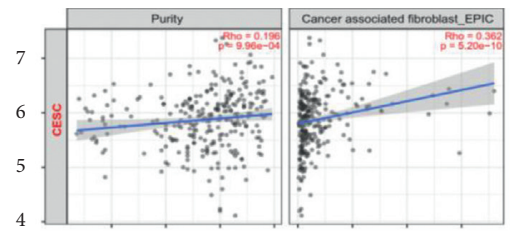

$\begin{array}{lllllll}0.25 & 0.50 & 0.75 & 1.000 .0 & 0.1 & 0.2 & 0.3\end{array}$
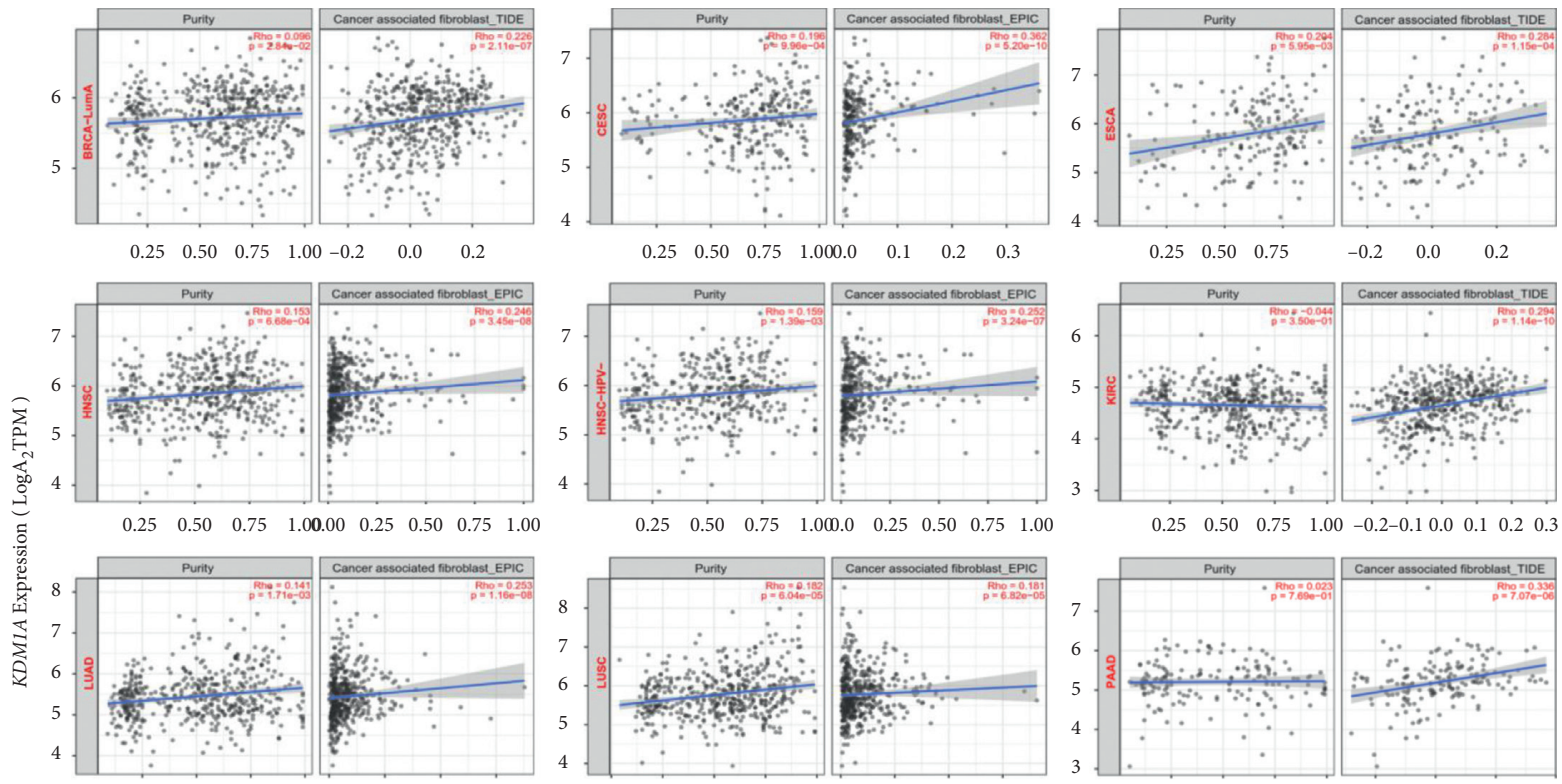

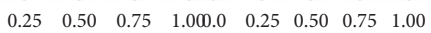

$\begin{array}{lllllllll}0.25 & 0.50 & 0.75 & 1.00 & -0.2-0.1 & 0.0 & 0.1 & 0.2 & 0.3\end{array}$
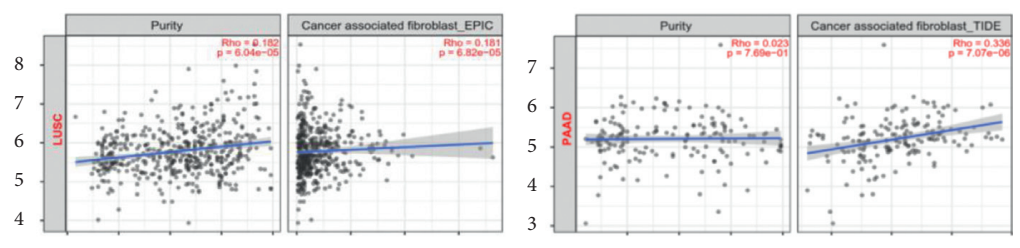

$\begin{array}{lllllll}0.25 & 0.50 & 0.75 & 1.00 .00 & 0.25 & 0.50 & 0.75\end{array}$

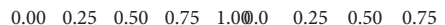

$\begin{array}{lllllll}0.25 & 0.50 & 0.75 & 1.00 & -0.2 & 0.0 & 0.2\end{array}$
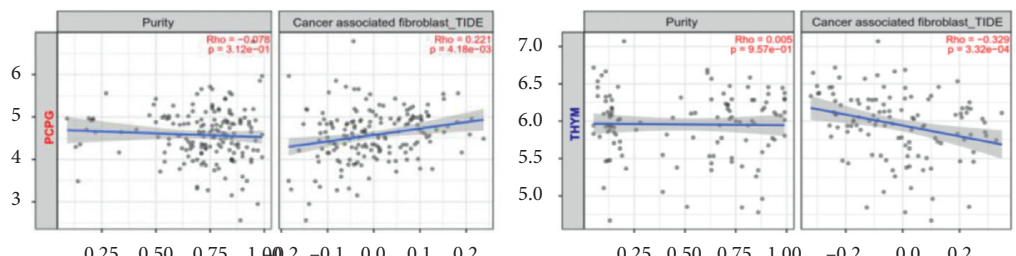

(b)

MDSC

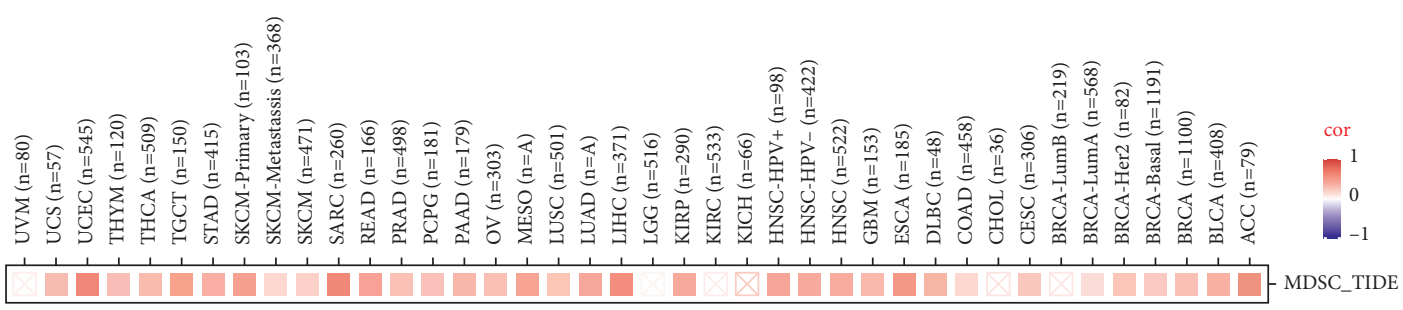

$\bigotimes p>0.05$

$\mathrm{p}<0.05$

(c)

FIGURE 5: Relationship of KDM1A with cancer-associated fibroblasts (CAFs) and myeloid-derived suppressor cells (MDSCs) in the tumor microenvironment. (a) The scores of CAF were associated with the expression of KDM1A gene via EPIC, MCPCOUNTER, and TIDE algorithms. (b) Correlation between KDM1A expression and infiltration level of CAFs. (c) TIDE algorithm showed MDSCs were positively correlated with KDM1A in most cancer types. 

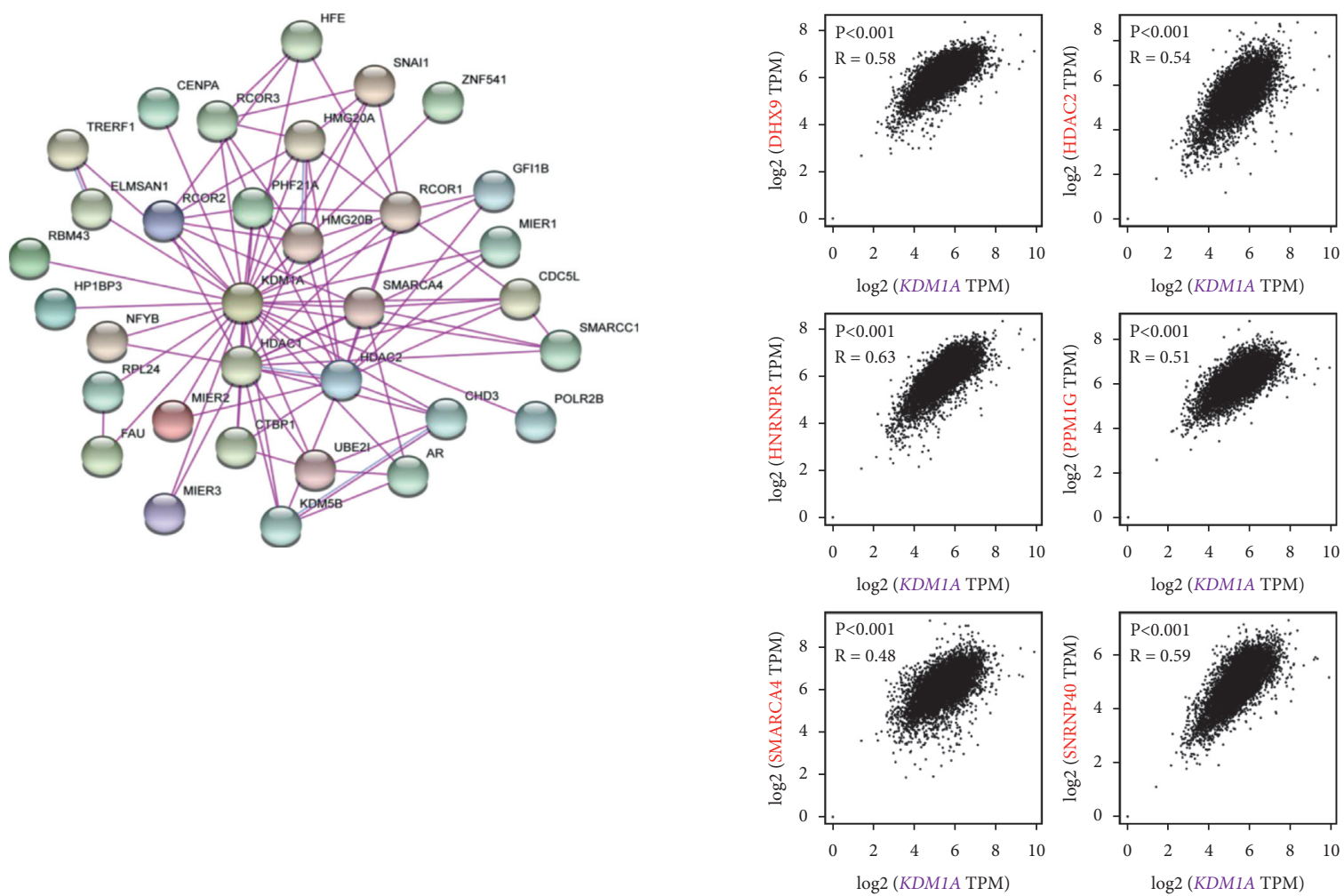

(a)
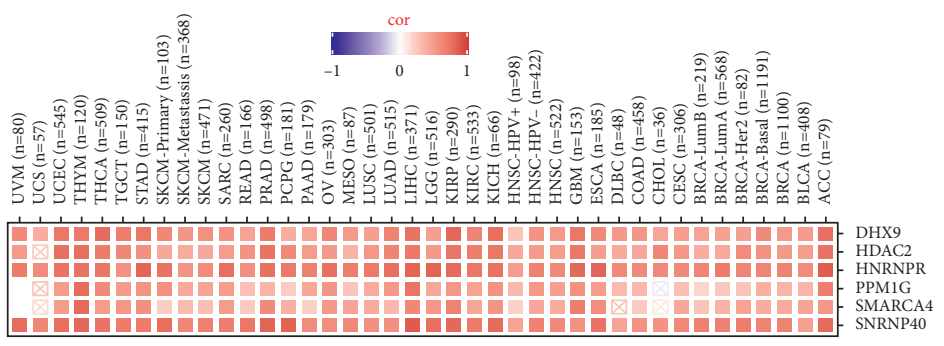

区 $P>0.05$

- $\mathrm{P}<0.05$

(b)

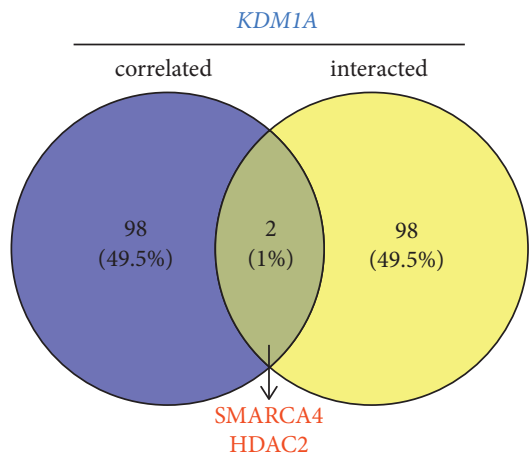

(c)

(d)

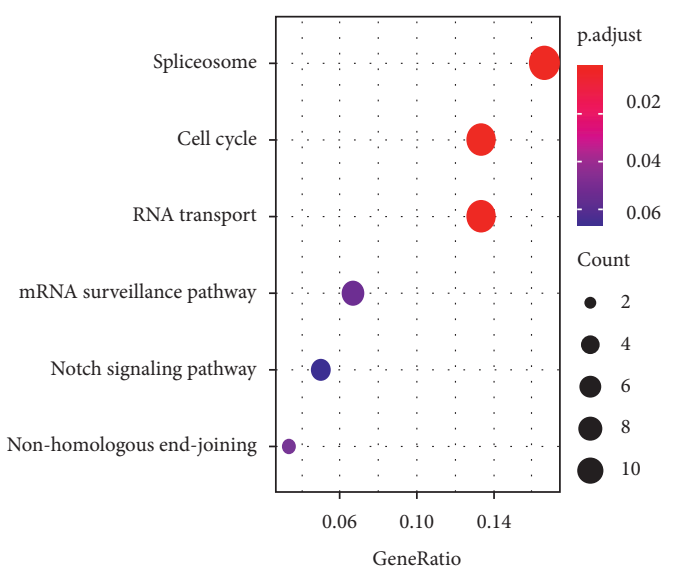

(e)

FIGURE 6: KDM1A-related gene enrichment analysis. (a) KDM1A-binding proteins were determined using the STRING tool. (b) The correlation of KDM1A and 6 top targeting genes was analyzed by GEPIA2. (c) The heatmap showed a corresponding relationship in the detailed cancer types. (d) An intersection analysis was conducted with the KDM1A-binding and correlated genes. (e) The bubble plot displayed KEGG pathway analysis based on the KDM1A-binding and interacted genes. 


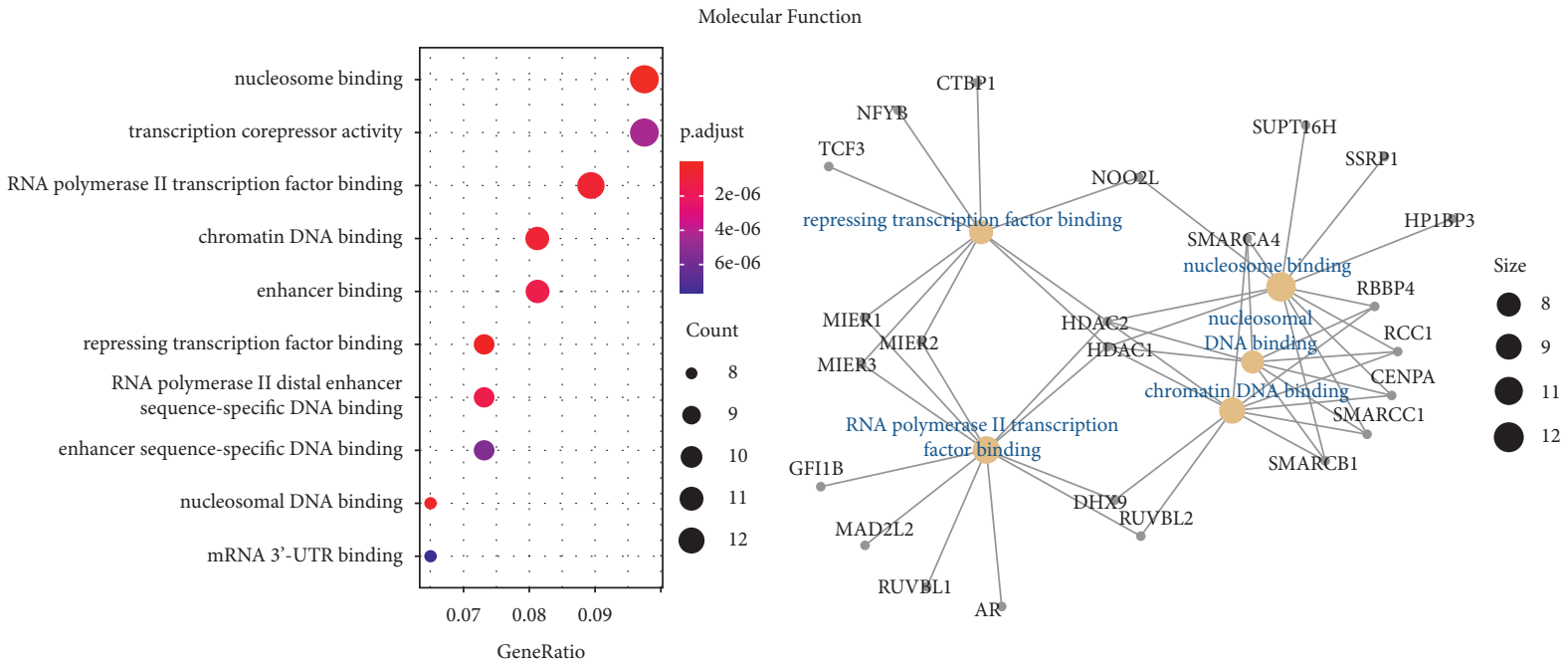

(a)
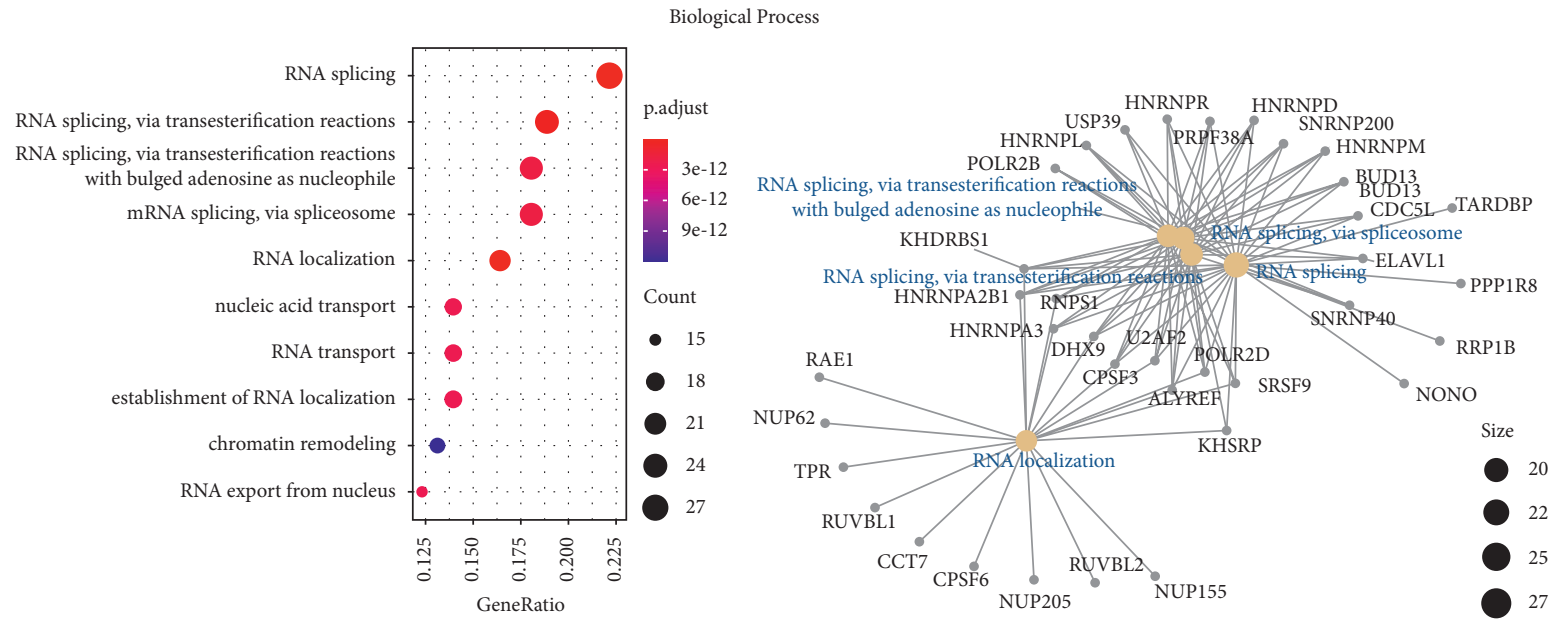

(b)
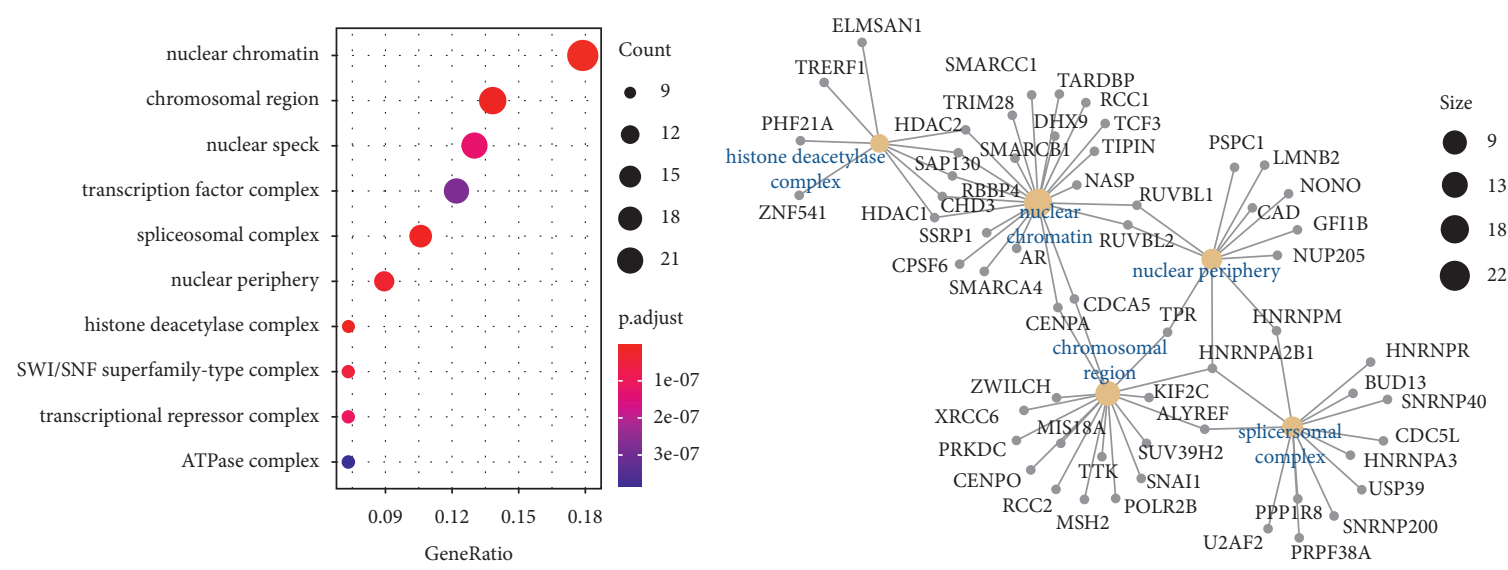

(c)

FIGURE 7: GO enrichment analysis of two gene sets referring to genes of KDM1A-binding and KDM1A-correlated genes. And the cnetplot for GO analysis of the first five was also shown: (a) molecular function analysis, (b) biological process analysis, and (c) cellular component analysis. 
In the present study, we compared the expression of KDM1A in 33 tumors and their corresponding normal tissues and found that KDM1A was differentially highly expressed in up to 21 tumors, and 17 types in them were highly expressed in tumors compared with normal tissues. Meanwhile, we explored whether KDM1A expression is related to survival prognosis. We found that in most tumors, the high expression of KDM1A was a risk factor and associated with poor OS and DFS. Furthermore, the survival analysis revealed that KDM1A in LIHC and LUAD was the high expression and associated with poor survival prognosis (Table 1). In addition, the mutation of KDM1A in BRCA exhibited poor survival, yet the high DNA methylation of KDM1A foreboded a better survival prognosis of breast cancer via decreasing KDM1A expression. Moreover, the phosphorylation levels of KDM1A were upregulated in breast cancer, UCEC, and LUAD, and the phosphorylation of KDM1A at S131 and S137 was experimentally supposed to play a role in regulating RNF168-dependent 53BP1 recruitment in response to DNA damage and resisting DNA damaging agents $[23,42]$. Meanwhile, Liu et al. showed that the overexpression of KDM1A is a potential prognostic factor in patients with liver cancer and KDM1A promotes tumorigenesis and malignancy in vitro [43]. Interestingly, high KDM1A expression in $\mathrm{KICH}$ was linked to poor prognosis, although it was the low expression in $\mathrm{KICH}$ compared with normal tissue from the TCGA database. Meanwhile, it has been reported that KDM1A can regulate kidney cancer cell growth via epigenetic control of AR transcription factors and that KDM1A inhibitors may be good candidate drugs for treating kidney cancer [44]. For UCEC cases, KDM1A is highly expressed, and the proportion of mutations is highest in all 33 tumors. Chen et al. demonstrated that silencing of KDM1A can abolish estrogen-driven endometrial cancer cell (ECC) proliferation and induce $\mathrm{G} 1$ cell arrest and apoptosis via PI3K/AKT/cyclinD1 signal [45]. These indicated that KDM1A is a potential prognostic biomarker in several cancers. Numerous KDM1A inhibitors had been discovered, and 8 of them had been used in clinical trials for multiple solid tumors and hematologic malignancy. Our result implied KDM1A inhibitors could have a potential effect on a wider spectrum of tumors, which can be further proved via experimental evidence.

Tumor microenvironment, including the immune and stromal microenvironment, constitutes a vital element of tumor tissue, which was closely related to oncogenesis and metastasis. Cancer-associated fibroblasts (CAFs) in the stroma participate in modulating the infiltration and function of various immune cells [46, 47]. Our analysis observed a statistically positive correlation between KDM1A expression and cancer-associated fibroblasts in most cancer types via multiple algorithms. Moreover, Liu and colleagues reported that upregulated KDM1A expression in CAFs is a driver of Notch3-mediated cancer stem-like cells self-renewal in hepatocellular carcinoma [43]. In addition, we illustrated that the positive correlation between KDM1A expression and MDSC infiltration happened in most cancers. MDSCs, as a heterogeneous group of myeloid cells, own potent immunosuppressive activity via interacting with innate and adaptive immune cells and perform a significant role in modulating antitumor immunity [48]. For adaptive immune cells, a statistically negative correlation was shown between KDM1A expression and $\mathrm{CD}^{8+} \mathrm{T}$ cell infiltration in TGCT, LGG, KIRP, KIRC, and HNSC-HPV ${ }^{+}$. KDM1A was negatively correlated with Th1 cells and B memory cells but positively correlated with Th2 cells in most cancer types. This implied KDM1A potentially related to immunosurveillance escape. Our previous study reported that KDM1A ablation stimulated tumor immunogenicity and increased T cell infiltration in breast cancer [7]. Sheng et al. also verified that LSD1 inhibition in tumor cells stimulated antitumor $\mathrm{T}$ cell immunity and overcame resistance to checkpoint blockade therapy [13]. These studies demonstrated that inhibition of KDM1A could increase the infiltration of $\mathrm{CD}^{8+} \mathrm{T}$ cells from different perspectives, which promoted the efficacy of immunotherapy. We suggested that KDM1A could become a new prognostic biomarker for antitumor immunotherapy, and the combination of KDM1A inhibitors and immunotherapy could exert a potent efficacy of tumor suppression.

In this study, we combined the KDM1A-binding components and KDM1A expression-related genes for downstream analyses and evaluated the potential roles of KDM1A on "cell cycle pathway," "RNA transport pathway," "DNA binding," and "RNA splicing." The intersection of KDM1Abinding components and KDM1A-related genes included HDAC2 and SMARCA4, which indicated the efficacy of KDM1A on cancer mainly through cooperating with other epigenetic regulatory factors to finely regulate downstream genes. It implied the combination therapy of multiple epigenetic inhibitors could increase synergy effect and safety.

Gut microbiota have been found to link with both local gastrointestinal cancers and other distal tumors [49]. Microbial metabolites were proved to regulate the development of cancer via epigenetic regulators, such as propionic and butyric acids $[49,50]$. Wang et al. demonstrated that the expression of KDM1A is upregulated by microbial metabolite butyrate in adipocytes [51]. It suggested that microbial metabolites may impact the KDM1A level in cancer cells to regulate tumor progression, which needs to be proved via experimental evidence.

Carcinogenic infections with certain viruses, bacteria, and parasites are strong risk factors for specific cancers [52]. KDM1A can impact viral and parasitic infections via the epigenetic regulation of viral genes and immune response. KDM1A activates replication of herpes simplex virus and varicella-zoster virus from latency via demethylating $\mathrm{H} 3 \mathrm{~K} 9$ at the viral immediate-early (IE) gene promoters [53]. KDM1A mediates the activation of the hepatitis $B$ virus via demethylating $\mathrm{H} 3 \mathrm{~K} 9$ and synergizing with Set1A methylating H3K4 [54]. On the other hand, Douce et al. reported that LSD1 cooperating with CTIP2 silences HIV-1 transcription and viral expression [55]. Furthermore, KDM1A downregulates PD-1 expression of CD8 T cells via histone H3K4 modification following acute viral infection [56]. KDM1A is also important for goblet cell maturation and effector responses of gut immunity to bacterial and helminth 
infections [57]. Meanwhile, KDM1A protects from endotoxin-induced death via regulating hematopoietic stem cells homeostasis [58]. The above studies show that KDM1A may have various effects on different types of infections.

\section{Conclusion}

Our comprehensive pan-cancer analysis illustrates the role of KDM1A as an oncogene and predictor of worse survival in most tumor types. KDM1A correlated with immunosuppressive tumor microenvironment via various approaches based on pan-cancer analysis. These findings highlight the role of KDM1A in tumorigenesis and development and potentially enable more precise and personalized immunotherapy in the future.

\section{Abbreviations}

ACC: Adrenocortical carcinoma

BLCA: Bladder urothelial carcinoma

BRCA: Breast invasive carcinoma

CESC: Cervical squamous cell carcinoma and endocervical adenocarcinoma

CHOL: Cholangiocarcinoma

COAD: Colon adenocarcinoma

DLBC: Lymphoid neoplasm diffuse large B-cell lymphoma

ESCA: Esophageal carcinoma

GBM: Glioblastoma multiforme

HNSC: Head and neck squamous cell carcinoma

$\mathrm{KICH}$ : Kidney chromophobe

KIRC: Kidney renal clear cell carcinoma

KIRP: Kidney renal papillary cell carcinoma

LAML: Acute myeloid leukemia

LGG: Brain lower grade glioma

LIHC: Liver hepatocellular carcinoma

LUAD: Lung adenocarcinoma

LUSC: Lung squamous cell carcinoma

MESO: Mesothelioma

OV: Ovarian serous cystadenocarcinoma

PAAD: Pancreatic adenocarcinoma

PCPG: Pheochromocytoma and paraganglioma

PRAD: Prostate adenocarcinoma

READ: Rectum adenocarcinoma

SARC: Sarcoma

SKCM: Skin cutaneous melanoma

STAD: Stomach adenocarcinoma

TGCT: Testicular germ cell tumors

THCA: Thyroid carcinoma

THYM: Thymoma

UCEC: Uterine corpus endometrial carcinoma

UCS: Uterine carcinosarcoma

UVM: Uveal melanoma

OS: Overall survival

DMFS: Distant metastasis-free survival

RFS: Relapse-free survival

PPS: Post-progression survival

FP: $\quad$ First progression

DSS: Disease-specific survival

PFS: Progression-free survival.

\section{Data Availability}

The data used to support the findings of this study are available from the corresponding authors upon request.

\section{Conflicts of Interest}

The authors declare that no competing interest exists.

\section{Authors' Contributions}

Y. Q. and H. W. conceived and supervised the study; L. L. and Y. W. performed data analyses; Y. M. helped in the analysis; all the authors contributed to drafting the manuscript; and Y. Q., L. L., and H. W. produced the final version of the manuscript.

\section{Acknowledgments}

This work was supported by grants from the Scientific Research Program of Hubei Education Department (D20201202) and Three Gorges University Master's Dissertation Training Fund (2021SSPY099).

\section{Supplementary Materials}

Survival analysis of Kaplan-Meier plotter. The Kaplan-Meier plotter (http://kmplot.com/analysis/) is a web-based tool of which aim is meta-analysis-based discovery and validation of survival biomarkers. The Kaplan-Meier plotter was used to analyze the correlations between KDM1A expression and patient survival of OS, DMFS (distant metastasis-free survival), RFS (relapse-free survival), PPS (post-progression survival), FP (first progression), DSS (disease-specific survival), and PFS (progress-free survival) in breast, ovarian, lung (LUAD and LUSC), gastric, and liver cancers. The data of breast, ovarian, lung (LUAD and LUSC), and gastric cancer came from gene chip (Affy ID: 212348_s_at (KDM1)), while one of liver cancer came from RNAseq (ID: 23028 (KDM1A)). The cases of these cancers were split into two groups by setting “autoselect best cutoff”. The hazard ratio (HR), 95\% confidence intervals, and log-rank $p$-value were computed, and the Kaplan-Meier survival plots were generated. Phosphorylation feature prediction. The openaccess PhosphoNET database ((http://www.phosphonet.ca/) was used to obtain the predicted phosphorylation features of the S69, S131, Y135, Y136, S137, S166, and S849 sites by searching the protein name "KDM1A". Figure S1: KDM1A expression in various cancers and pathological stages. (a) The expression levels of KDM1A gene in different cancers from TCGA were compared with the corresponding normal tissues based on GTEx databases. (b) KDM1A expression in different pathological stages in selected cancer types. Figure S2: Survival prognosis of cancers was related to the expression of KDM1A analyzed by the GEPIA2 tool. Figure S3: Correlation between KDM1A gene expression and prognosis of cancers. The Kaplan-Meier plot showed the survival curve by comparison of the cases with high and low expression of KDM1A in breast cancer (a), ovarian cancer (b), gastric cancer (c), LUAD (d), LUSC (e), and liver cancer (f) 
and the curves were plotted from the Kaplan-Meier plotter database. OS, overall survival; DMFS, distant metastasis-free survival; RFS, relapse-free survival; PPS, post-progression survival; FP, first progression; DSS, disease-specific survival; PFS, progress-free survival. The data of breast, ovarian, lung (LUAD and LUSC), and gastric cancer came from gene chip (Affy ID: 212348_s_at (KDM1)), while one of liver cancer came from RNAseq (ID: 23028 (KDM1A)). Figure S4: Correlation between DNA methylation of KDM1A and survival prognosis in TCGA tumors using MethSurv. We used the MethSurv website to perform multivariable survival analysis using DNA methylation data. The $p$ value $(<0.05)$ and the hazard ratio (HR) are displayed. Figure S5: Phosphorylation level of KDM1A protein (NP_0055828.2) in different tumors based on the CPTAC data set, including ovarian cancer (a), UCEC (b), LUAD (c), and colon cancer (d). Figure S6: Correlation between KDM1A expression and $\mathrm{CD}^{8+} \mathrm{T}$ cell infiltration across all types of cancer in TCGA based on different algorithms. Figure S7: Correlation between KDM1A expression and the infiltration of $\mathrm{CD}^{4+}$ T cells, Tregs, and B cells across all types of cancer in TCGA based on different algorithms. (a) $\mathrm{CD}^{4+} \mathrm{T}$ cells, (b) Tregs, and (c) B cells. Figure S8: Correlation between KDM1A expression and the infiltration of myeloid-derived cells across all types of cancer in TCGA based on different algorithms. (a) Macrophage, (b) myeloid dendritic cell, and (c) neutrophil. Table S1: Alteration sites of KDM1A in tumors. Table S2: Phosphorylation level of KDM1A protein in different tumors. Table S3: Analysis of CPTAC-identified phosphorylation sites of KDM1A via the PhosphoNET database. (Supplementary Materials)

\section{References}

[1] S. Biswas and C. M. Rao, "Epigenetics in cancer: fundamentals and beyond," Pharmacology \& Therapeutics, vol. 173, pp. 118-134, 2017.

[2] S. J. Hogg, P. A. Beavis, M. A. Dawson, and R. W. Johnstone, "Targeting the epigenetic regulation of antitumour immunity," Nature Reviews Drug Discovery, vol. 19, no. 11, pp. 776-800, 2020.

[3] Y. Shi, F. Lan, C. Matson et al., "Histone demethylation mediated by the nuclear amine oxidase homolog LSD1," Cell, vol. 119, no. 7, pp. 941-953, 2004.

[4] E. Metzger, "LSD1 demethylates repressive histone marks to promote androgen-receptor-dependent transcription," $\mathrm{Na}$ ture, vol. 437, pp. 436-439, 2005.

[5] J. Wang, "LSD1n is an H4K20 demethylase regulating memory formation via transcriptional elongation control," Nature Neuroscience, vol. 18, pp. 1256-1264, 2015.

[6] D. Kim, K. I. Kim, and S. H. Baek, "Roles of lysine-specific demethylase 1 (LSD1) in homeostasis and diseases," Journal of Biomedical Science, vol. 28, p. 41, 2021.

[7] Y. Qin, "Inhibition of histone lysine-specific demethylase 1 elicits breast tumor immunity and enhances antitumor efficacy of immune checkpoint blockade," Oncogene, vol. 38, pp. 390-405, 2019.

[8] Q. Xie, "LSD1 promotes bladder cancer progression by upregulating LEF1 and enhancing EMT," Front Oncol, vol. 10, p. 1234, 2020.
[9] S. Gao, "Chromatin binding of FOXA1 is promoted by LSD1mediated demethylation in prostate cancer," Nature Genetics, vol. 52, pp. 1011-1017, 2020.

[10] J. Wang, "Novel histone demethylase LSD1 inhibitors selectively target cancer cells with pluripotent stem cell properties," Cancer Research, vol. 71, pp. 7238-7249, 2011.

[11] J. Y. Lee, "LSD1 demethylates HIF1 $\alpha$ to inhibit hydroxylation and ubiquitin-mediated degradation in tumor angiogenesis," Oncogene, vol. 36, pp. 5512-5521, 2017.

[12] T. Lin, A. Ponn, X. Hu, B. K. Law, and J. Lu, "Requirement of the histone demethylase LSD1 in Snail-mediated transcriptional repression during epithelial-mesenchymal transition," Oncogene, vol. 29, pp. 4896-4904, 2010.

[13] W. Sheng, "LSD1 ablation stimulates anti-tumor immunity and enables checkpoint blockade," Cell, vol. 174, pp. 549-563, 2018.

[14] X. Cui, "A pan-cancer analysis of the oncogenic role of staphylococcal nuclease domain-containing protein 1 (SND1) in human tumors," Genomics, vol. 112, pp. 3958-3967, 2020.

[15] T. Li, J. Fu, Z. Zeng et al., "TIMER2.0 for analysis of tumorinfiltrating immune cells," Nucleic Acids Research, vol. 48, pp. W509-w514, 2020.

[16] Z. Tang, B. Kang, C. Li, T. Chen, and Z. Zhang, "GEPIA2: an enhanced web server for large-scale expression profiling and interactive analysis," Nucleic Acids Research, vol. 47, pp. W556-w560, 2019.

[17] D. S. Chandrashekar, B. Bashel, S. A. H. Balasubramanya et al., "UALCAN: a portal for facilitating tumor subgroup gene expression and survival analyses," Neoplasia, vol. 19, pp. 649-658, 2017.

[18] E. Cerami, "The cBio cancer genomics portal: an open platform for exploring multidimensional cancer genomics data," Cancer Discovery, vol. 2, pp. 401-404, 2012.

[19] V. Modhukur, T. Iljasenko, T. Metsalu, K. Lokk, T. LaiskPodar, and J. Vilo, "MethSurv: a web tool to perform multivariable survival analysis using DNA methylation data," Epigenomics, vol. 10, pp. 277-288, 2018.

[20] A. Koch, J. Jeschke, W. Van Criekinge, M. van Engeland, and T. De Meyer, "MEXPRESS update 2019," Nucleic Acids Research, vol. 47, pp. W561-W565, 2019.

[21] D. Szklarczyk, "STRING v11: protein-protein association networks with increased coverage, supporting functional discovery in genome-wide experimental datasets," Nucleic Acids Research, vol. 47, pp. D607-d613, 2019.

[22] G. Yu, L. G. Wang, Y. Han, and Q. Y. He, "clusterProfiler: an R package for comparing biological themes among gene clusters," Omics, vol. 16, pp. 284-287, 2012.

[23] B. Peng, "Modulation of LSD1 phosphorylation by CK2/WIP1 regulates RNF168-dependent 53BP1 recruitment in response to DNA damage," Nucleic Acids Research, vol. 43, pp. 59365947, 2015.

[24] L. Chen, "Functional characterization of lysine-specific demethylase 2 (LSD2/KDM1B) in breast cancer progression," Oncotarget, vol. 8, pp. 81737-81753, 2017.

[25] B. Majello, F. Gorini, C. D. Saccà, and S. Amente, "Expanding the role of the histone lysine-specific demethylase LSD1 in cancer," Cancers (Basel), vol. 11, 2019.

[26] F. Gu, Y. Lin, Z. Wang et al., "Biological roles of LSD1 beyond its demethylase activity," Cellular and Molecular Life Sciences, vol. 77, pp. 3341-3350, 2020.

[27] M. G. Lee, C. Wynder, N. Cooch, and R. Shiekhattar, "An essential role for CoREST in nucleosomal histone 3 lysine 4 demethylation," Nature, vol. 437, pp. 432-435, 2005.

[28] Y. Shi, "Coordinated histone modifications mediated by a CtBP co-repressor complex," Nature, vol. 422, pp. 735-738, 2003. 
[29] Y. Wang, "LSD1 is a subunit of the NuRD complex and targets the metastasis programs in breast cancer," Cell, vol. 138, pp. 660-672, 2009.

[30] B. Laurent, "A specific LSD1/KDM1A isoform regulates neuronal differentiation through H3K9 demethylation," Molecular Cell, vol. 57, pp. 957-970, 2015.

[31] J. Wang, "Opposing LSD1 complexes function in developmental gene activation and repression programmes," Nature, vol. 446 , pp. 882-887, 2007.

[32] D. N. Ciccone, "KDM1B is a histone H3K4 demethylase required to establish maternal genomic imprints," Nature, vol. 461, pp. 415-418, 2009.

[33] T. A. Katz, S. N. Vasilatos, E. Harrington, S. Oesterreich, N. E. Davidson, and Y. Huang, "Inhibition of histone demethylase, LSD2 (KDM1B), attenuates DNA methylation and increases sensitivity to DNMT inhibitor-induced apoptosis in breast cancer cells," Breast Cancer Research and Treatment, vol. 146, pp. 99-108, 2014.

[34] Y. K. Lee, J. Lim, S. Y. Yoon, J. C. Joo, S. J. Park, and Y. J. Park, "Promotion of cell death in cisplatin-resistant ovarian cancer cells through KDM1B-DCLRE1B modulation," International Journal of Molecular Sciences, vol. 20, 2019.

[35] Y. Wang, L. Sun, Y. Luo, and S. He, "Knockdown of KDM1B inhibits cell proliferation and induces apoptosis of pancreatic cancer cells," Pathology, Research \& Practice, vol. 215, pp. 1054-1060, 2019.

[36] A. Maiques-Diaz, "Enhancer activation by pharmacologic displacement of LSD1 from GFI1 induces differentiation in acute myeloid leukemia," Cell Reports, vol. 22, pp. 3641-3659, 2018.

[37] W. A. Whyte, S. Bilodeau, D. A. Orlando et al., "Enhancer decommissioning by LSD1 during embryonic stem cell differentiation," Nature, vol. 482, pp. 221-225, 2012.

[38] S. Ambrosio, A. Ballabio, and B. Majello, "Histone methyltransferases and demethylases in the autophagy regulatory network: the emerging role of KDM1A/LSD1 demethylase," Autophagy, vol. 15, pp. 187-196, 2019.

[39] J. Castex, "Inactivation of Lsd1 triggers senescence in trophoblast stem cells by induction of Sirt4," Cell Death \& Disease, vol. 8, Article ID e2631, 2017.

[40] M. A. Christopher, "LSD1 protects against hippocampal and cortical neurodegeneration," Nature Communications, vol. 8, p. 805, 2017.

[41] A. Sakamoto, "Lysine demethylase LSD1 coordinates glycolytic and mitochondrial metabolism in hepatocellular carcinoma cells," Cancer Research, vol. 75, pp. 1445-1456, 2015.

[42] R. Costa, G. Arrigoni, G. Cozza et al., "The lysine-specific demethylase 1 is a novel substrate of protein kinase CK2," Biochimica et Biophysica Acta, vol. 1844, pp. 722-729, 2014.

[43] C. Liu, "LSD1 stimulates cancer-associated fibroblasts to drive notch3-dependent self-renewal of liver cancer stem-like cells," Cancer Research, vol. 78, pp. 938-949, 2018.

[44] K. H. Lee, B. C. Kim, S. H. Jeong et al., "Histone demethylase LSD1 regulates kidney cancer progression by modulating androgen receptor activity," International Journal of Molecular Sciences, vol. 21, 2020.

[45] C. Chen, "LSD1 sustains estrogen-driven endometrial carcinoma cell proliferation through the PI3K/AKT pathway via di-demethylating H3K9 of cyclin D1," International Journal of Oncology, vol. 50, pp. 942-952, 2017.

[46] M. Paauwe, "Endoglin expression on cancer-associated fibroblasts regulates invasion and stimulates colorectal cancer metastasis," Clinical Cancer Research, vol. 24, pp. 6331-6344, 2018.
[47] X. Chen and E. Song, "Turning foes to friends: targeting cancer-associated fibroblasts," Nature Reviews Drug Discovery, vol. 18, pp. 99-115, 2019.

[48] C. Zalfa and S. Paust, "Natural killer cell interactions with myeloid derived suppressor cells in the tumor microenvironment and implications for cancer immunotherapy," Frontiers in Immunology, vol. 12, p. 633205, 2021.

[49] S. Vivarelli, "Gut microbiota and cancer: from pathogenesis to therapy," Cancers (Basel), vol. 11, 2019.

[50] W. S. Garrett, "Cancer and the microbiota," Science, vol. 348, pp. 80-86, 2015.

[51] D. Wang, "LSD1 mediates microbial metabolite butyrateinduced thermogenesis in brown and white adipose tissue," Metabolism, vol. 102, p. 154011, 2020.

[52] M. Plummer, C. de Martel, J. Vignat, J. Ferlay, F. Bray, and S. Franceschi, "Global burden of cancers attributable to infections in 2012: a synthetic analysis," Lancet Glob Health, vol. 4, pp. e609-16, 2016.

[53] Y. Liang, D. Quenelle, J. L. Vogel, C. Mascaro, A. Ortega, and T. M. Kristie, "A novel selective LSD1/KDM1A inhibitor epigenetically blocks herpes simplex virus lytic replication and reactivation from latency," mBio, vol. 4, pp. e00558-12, 2013.

[54] V. Alarcon, "The enzymes LSD1 and Set1A cooperate with the viral protein $\mathrm{HBx}$ to establish an active hepatitis B viral chromatin state," Scientific Reports, vol. 6, Article ID 25901, 2016.

[55] V. Le Douce, "LSD1 cooperates with CTIP2 to promote HIV1 transcriptional silencing," Nucleic Acids Research, vol. 40, pp. 1904-1915, 2012.

[56] A. P. R. Bally, D. K. Neeld, P. Lu et al., "PD-1 expression during acute infection is repressed through an LSD1-blimp-1 Axis," The Journal of Immunology, vol. 204, pp. 449-458, 2020.

[57] N. Parmar, "Intestinal-epithelial LSD1 controls goblet cell maturation and effector responses required for gut immunity to bacterial and helminth infection," PLoS Pathogens, vol. 17, Article ID e1009476, 2021.

[58] J. Wang, K. Saijo, D. Skola et al., "Histone demethylase LSD1 regulates hematopoietic stem cells homeostasis and protects from death by endotoxic shock," Proceedings of the National Academy of Sciences of the USA, vol. 115, pp. E244-e252, 2018. 